\title{
Pouvoir et propriété dans l'entreprise Pour une histoire internationale des sociétés à responsabilité limitée*
}

\author{
Timothy W. Guinnane, Ron Harris, \\ Naomi R. Lamoreaux et Jean-Laurent Rosenthal
}

Les liens entre I'histoire du capitalisme et l'avènement des sociétés anonymes sont profonds. L'histoire du capitalisme industriel ou du capitalisme financier se déploie la plupart du temps autour des grandes entreprises: or ces hérö̈nes de la transformation économique n'auraient pu voir le jour sans une structure légale permettant d'en pérenniser le capital, d'en déléguer la gérance et de limiter la responsabilité des investisseurs. La transition vers le capitalisme a ainsi dépendu d'une mutation du droit qu'il est courant de réduire à la création des sociétés anonymes. Cette conception très répandue est venue conforter deux a priori. La société anonyme dans sa forme idéale (faible coût de création, gérance concentrée mais choisie par un conseil d'administration élu, responsabilité limitée et libre circulation des actions) est perçue comme la meilleure façon d'organiser une entreprise. Et, comme les sociétés anonymes les plus proches de cette forme idéale se

\footnotetext{
* Nous sommes reconnaissants à Svetlana Alkayeva, Ofira Alon, Juan-Francisco Aveleyra, Christopher Cook, Sarah Cullem, Olga Frishman, Theresa Gutberlet, Adam Hofri, Alena Laptiovna, Maria Polyakova, Itai Rabinowitz, Olga Sedjakina, Sarah Shen, Yvonne Taylor, et Eyal Yaacoby pour leur aide. Nous voudrions aussi remercier les institutions suivantes pour leur soutien financier: le McMillan International Studies Center et le Millstein Center for Corporate Governance and Performance à Yale University, International Institute, le doyen des sciences sociales à UCLA, UCLA Academic Senate, le Center for Advanced Study in the Behavioral Sciences à Stanford, l'Israel Science Foundation et l'École d'économie de Paris. Nous remercions de même Gary Herrigel, Leslie Hannah, Henry Hansmann, Eric Hilt, Timur Kuran, Jonathan Macey, Roberta Romano, Otto Scherner, Kenneth Sokoloff, Jochen Streb, et tout particulièrement Gilles Postel-Vinay pour leurs conseils et critiques.
} 
sont largement diffusées aux États-Unis et au Royaume-Uni, on en déduit que les institutions légales anglo-américaines auraient offert aux entrepreneurs un meilleur contexte que celles de l'Europe. D'une certaine façon, ces postulats furent le produit du siècle américain : celui pendant lequel l'économie des États-Unis a été dominante et a été dominée par les sociétés anonymes.

Nous souhaitons nous inscrire en faux contre cette simplification des liens entre le droit de l'entreprise et l'évolution des économies. Si les entreprises pluripropriétaires n'ont cessé de s'étendre en nombre et en taille, elles ont été sujettes à des problèmes de gouvernance persistants et la société anonyme n'est pas une panacée. Même aux États-Unis, la plupart des sociétés sont restées des sociétés en nom collectif ( $\mathrm{SNC}$ ) au moins un siècle après le vote des lois libéralisant l'accès aux sociétés anonymes (SA). À la fin du $\mathrm{XIX}^{\mathrm{e}}$ et au début du XX $\mathrm{XX}^{\mathrm{e}}$ siècle, les pays européens ont créé des formes alternatives que nous appellerons ici des sociétés à responsabilité limitée (SARL). Chaque fois qu'une telle forme a été offerte aux entrepreneurs (en Allemagne avec la GmbH en 1892, en Angleterre avec la PLLC en 1908, et en France en 1925), elle est rapidement devenue beaucoup plus populaire que la société anonyme. À cet égard, la situation des États-Unis est singulière puisque les entreprises n'ont pas vraiment eu accès à ce type de société avant la seconde moitié du $\mathrm{XX}^{\mathrm{e}}$ siècle.

Bien que les caractéristiques légales des SARL varient d'un pays à l'autre, ce sont toutes des sociétés par actions, avec une responsabilité limitée des investisseurs ${ }^{1}$. Elles ne peuvent faire appel public à l'épargne et leurs actions ne peuvent pas être cotées mais, en contrepartie, toutes bénéficient de faibles charges d'administration et leurs obligations d'information sont réduites. L'accès à la responsabilité limitée a sans doute constitué un privilège suffisant pour expliquer que les entrepreneurs ont préféré les SARL aux sociétés en nom collectif, et la légèreté des obligations réglementaires a sans doute été la raison pour laquelle ils ont choisi des SARL plutôt que des sociétés anonymes. Nous montrerons cependant que, si importantes que soient ces caractéristiques, elles ne suffisent pas à expliquer la rapidité de la diffusion de ce type de société. En fait, le succès des SARL vient de ce qu'elles permettaient d'étendre l'éventail des structures de gouvernance d'entreprise. De plus, leur impact précis dépendait du contexte spécifique dans lequel la nouvelle forme a été introduite. Ainsi l'histoire du droit et de la législation des autres formes de société a toute son importance pour expliquer la popularité des SARL.

Cet argument se fonde sur une autre vision des choix d'organisation selon laquelle les associés cherchent un équilibre entre différents problèmes contractuels ${ }^{2}$. D’un côté, la société anonyme résout le principal désavantage des sociétés en nom collectif: celui d'être dissoutes trop facilement - nous parlerons de son aptitude à protéger l'entité de l'entreprise. De l'autre, sa structure hiérarchique présente

1 - La liste des différentes formes d'entreprise est donnée en annexe.

2 - Cet article est centré sur les choix que font les associés dans des entreprises pluripropriétaires, laissant de côté les questions relatives aux règles légales qui gouvernent les entreprises uninominales. 
l'inconvénient de permettre aux gérants de détourner une partie des profits au détriment des actionnaires. Si l'on veut éviter les coûts induits par une gérance pillarde, il faut soit modifier les articles de la société à un tel point que les attributs de la société anonyme risquent d'être perdus, soit choisir une autre forme d'organisation. Comme les propriétaires des plus grosses entreprises désirent que leurs investissements soient liquides, c'est-à-dire facilement négociables, ils organisent des sociétés anonymes. Mais la plupart des petites et moyennes entreprises (PME) n'ont que peu de propriétaires et leurs actions ne sont jamais cotées; il est donc possible que les entrepreneurs soient moins enclins à subir les désagréments des sociétés anonymes. Au XIX ${ }^{\mathrm{e}}$ siècle, les propriétaires de PME aux États-Unis ou au Royaume-Uni qui voulaient éviter ce problème ne pouvaient le faire qu'en formant des sociétés en nom collectif. En France et en Allemagne il existait déjà une troisième voie, la commandite, qui permettait une plus grande diversité de choix. L'avantage des SARL a été de donner aux entrepreneurs une grande latitude pour mélanger les attributs des sociétés anonymes et des sociétés en nom collectif.

Cet article fait partie d'un projet plus vaste portant sur l'impact de l'environnement juridique sur les sociétés, qu'elles soient cotées ou non. Bien que les travaux récents sur les liens entre droit et finance aient privilégié l'étude des sociétés cotées, l'environnement légal pèse encore plus sur le succès des PME dont les moyens ne permettent pas de payer avocats, intermédiaires financiers ou divers fournisseurs d'information. Nous montrerons que les SARL ont eu des conséquences bénéfiques importantes pour les PME et nous retracerons leur émergence puis leur diffusion en Allemagne, au Royaume-Uni, en France et aux ÉtatsUnis au cours des deux derniers siècles, avant d'explorer différentes hypothèses pour expliquer leur inégal succès. Pour ce faire, nous reconstruirons l'historique des SARL pays par pays, nous proposerons différentes façons de mesurer le rythme auquel elles ont été adoptées ainsi que leur impact sur l'utilisation des formes plus traditionnelles (SA, SNC). Enfin nous montrerons qu'investisseurs et entrepreneurs ont su tirer parti de la flexibilité des SARL. Durant la période considérée, les pays étudiés ont connu une croissance économique à long terme et furent les grands innovateurs en matière de droit. Les codes civils et commerciaux français et allemands forment la base du droit commercial dans de nombreux autres pays; la common law est née en Angleterre; et la démocratisation des sociétés anonymes est venue des États-Unis (autre pays de common law). Suivre ces quatre pays sur deux siècles suppose de surmonter certains obstacles car leurs différences rendent la collecte de données comparables ardue et parfois impossible ${ }^{3}$ mais notre approche comparative a un double avantage. Elle permet non seulement de s'affranchir des graves

3 - Par exemple, on dispose de très bonnes données sur le nombre d'entreprises qui choisissent différents statuts à partir de 1887 en Allemagne et de 1916 aux États-Unis, suite à la mise en place de l'impôt sur le revenu, mais elles n'apparaissent en France qu'en 1949. L'obligation d'enregistrer les statuts des entreprises auprès d'une autorité locale nous donne de très bonnes données sur les nouvelles entreprises en France et en Allemagne sur une période longue alors qu'aux États-Unis et au Royaume-Uni, il est possible de suivre les flux de sociétés anonymes mais pas ceux des sociétés en nom collectif qui, étant des entités de la common law, n'étaient soumises à aucune formalité. 
faiblesses qui entachent les analyses de l'évolution légale des formes d'entreprises menées pays par pays, mais aussi de contester l'idée fausse selon laquelle le droit commercial des États-Unis ou du Royaume-Uni devrait être le mètre étalon toujours et partout.

\section{Les avantages des SARL}

L'a priori qui veut que la société anonyme soit la meilleure forme d'entreprise découle de l'idée qu'elle seule permet l'accumulation intensive du capital (en particulier du capital fixe) nécessaire à la croissance économique ${ }^{4}$. À l'inverse, il est courant d'admettre que la société en nom collectif présente des défauts: elle serait trop instable pour créer les conditions favorables à des investissements sur le long terme. Parce que les sociétés en nom collectif sont dissoutes de droit par la mort d'un associé et que la jurisprudence a établi que leur activité doit être soumise à l'accord unanime des associés, le risque d'une liquidation à perte de ce type d'entreprise est élevé, ce qui accroît les réticences des associés avant d'engager des investissements spécifiques ou difficiles à liquider ${ }^{5}$. Elles ont aussi d'autres désavantages: une responsabilité sans limite, une gérance collective et une faible capacité à protéger l'entreprise des activités de ses propriétaires ${ }^{6}$.

Si la supériorité des sociétés anonymes était réelle, elles auraient dû supplanter les sociétés en nom collectif dès l'adoption des lois les libéralisant. Or en 1900, elles n'étaient majoritaires dans aucun pays. Le succès persistant des sociétés en nom collectif résulterait-il d'un avantage juridique vis-à-vis des sociétés anonymes, victimes de clauses restrictives ou de règles d'enregistrement défavorables ${ }^{7}$ ? À

\footnotetext{
4 - Alfred D. Chandler Jr., The visible hand: The managerial revolution in American business, Cambridge, Belknap Press, 1977; Nathan Rosenberg et Luther E. Birdzell Jr., How the West grew rich: The economic transformation of the industrial world, New York, Basic Books, 1986.

5 - Margaret M. BLAIR, «Locking in capital: What corporate law achieved for business organizers in the nineteenth century», UCLA Law Review, 51, 2003, p. 387-455; Larry E. RibSTEIN, «Why corporations? », Berkeley Business Law Journal, 1, 2004, p. 183-232. 6 - Sur la responsabilité limitée, voir Susan E. WoOdward, «Limited liability in the theory of the firm ", Journal of Institutional and Theoretical Economics, 141, 1985, p. 601611. Sur la protection des entités, voir Henry Hansmann, Reinier Kraakman et Richard SQuire, "Law and the rise of the firm ", Harvard Law Review, 119, 2006, p. 1333-1403. Pour une discussion théorique de l'inefficacité des gérances collectives, voir Armen A. Alchian et Harold Demsetz, «Production, information costs, and economic organization", American Economic Review, 62, 1972, p. 777-795; Oliver D. HART, Firms, contracts, and financial structure, Oxford/New York, Clarendon Press/Oxford University Press, 1995.

7 - De nos jours, la fiscalité joue un rôle majeur sur les choix des formes d'organisation. Mais avant le $\mathrm{XX}^{\mathrm{e}}$ siècle, la fiscalité était modérée et les éventuelles différences dans le traitement fiscal des sociétés anonymes par rapport aux sociétés en nom collectif étaient mineures. Nous avons donc choisi de laisser ces questions de côté.
} 
l'examen, il n'y a qu'en Allemagne, et seulement après 1884, que de telles interventions ont pesé contre la diffusion des sociétés anonymes.

Il est en fait plus plausible que la société anonyme n'ait pas été aussi pratique qu'on a pu le croire. Ses deux grands avantages (gérance concentrée et durée quasi perpétuelle) ne sont pas sans contrepartie ${ }^{8}$. Parce que les seuls membres à posséder le pouvoir dans l'entreprise sont ceux qu'élisent les actionnaires, toute coalition qui contrôle l'élection des dirigeants contrôle la firme. Si la coalition se sert de son pouvoir au détriment des autres actionnaires, les minoritaires ne disposent presque d'aucun recours: ils ne peuvent ni obliger la majorité à changer de politique ni ordonner une dissolution de l'entreprise afin de retrouver leur mise. Le problème est particulièrement grave dans les sociétés anonymes ayant peu de propriétaires, car ces derniers ne peuvent alors se retirer en vendant leurs parts.

Organiser une entreprise en société anonyme plutôt qu'en société en nom collectif revient à choisir entre deux formes imparfaites et deux types d'inconvénients : l'incapacité d'une société en nom collectif à se prémunir contre une dissolution prématurée (nous parlerons dorénavant du problème de dissolution prématurée) d'un côté, la possibilité d'avoir à faire face aux malversations des actionnaires dominants, risque principal dans une société anonyme (nous parlerons dorénavant du problème de l'oppression des minoritaires) de l'autre. Dans un monde où n'existent que deux types de sociétés, on s'attendrait donc à ce qu'elles coexistent.

Si l'argument est correct, l'avantage des SARL serait d'ouvrir l'éventail des choix, les propriétaires d'entreprise pouvant dès lors mélanger différents aspects des sociétés anonymes et des sociétés en nom collectif et ainsi mieux balancer risque de dissolution prématurée et risque d'oppression des minoritaires. La SARL n'a certes pas toujours les mêmes attributs selon les pays, mais partout elle offre une grande flexibilité contractuelle. Les entrepreneurs ont pu inclure dans les statuts de l'entreprise des clauses permettant d'affiner l'équilibre entre le risque d'oppression et celui de se trouver dans une impasse, ou bien encore entre le risque de dissolution prématurée et celui de devoir accepter une position d'otage. Pour amener les associés à réaliser des investissements spécifiques, par exemple, ils pouvaient rendre la dissolution difficile. Ou bien protéger les propriétaires minoritaires qui avaient fait de tels investissements en leur donnant un droit de veto dans certaines circonstances. De plus, cette nouvelle flexibilité n'impliquait pas l'abandon des avantages importants de la société anonyme, tels que la responsabilité limitée et la protection de l'entité de l'entreprise. Notre argument peut être illustré avec le graphique 1.

8 - Sur ce point, voir Naomi R. LAmoreaux et Jean-Laurent RosenthaL, "Corporate governance and the plight of minority shareholders in the United States before the Great Depression », in E. L. GLAEser et C. Goldin (éd.), Corruption and reform: Lessons from America's economic history, Chicago, University of Chicago Press, 2006, p. 125-152; Id., "Contractual tradeoffs and SMEs' choice of organizational form: A view from US and French history, 1830-2000 », working paper $n^{\circ} 12455$, National Bureau of Economic Research, Inc., 2006. 
Graphique 1 - Impact d'une loi SARL quand la formation des SA est facilitée

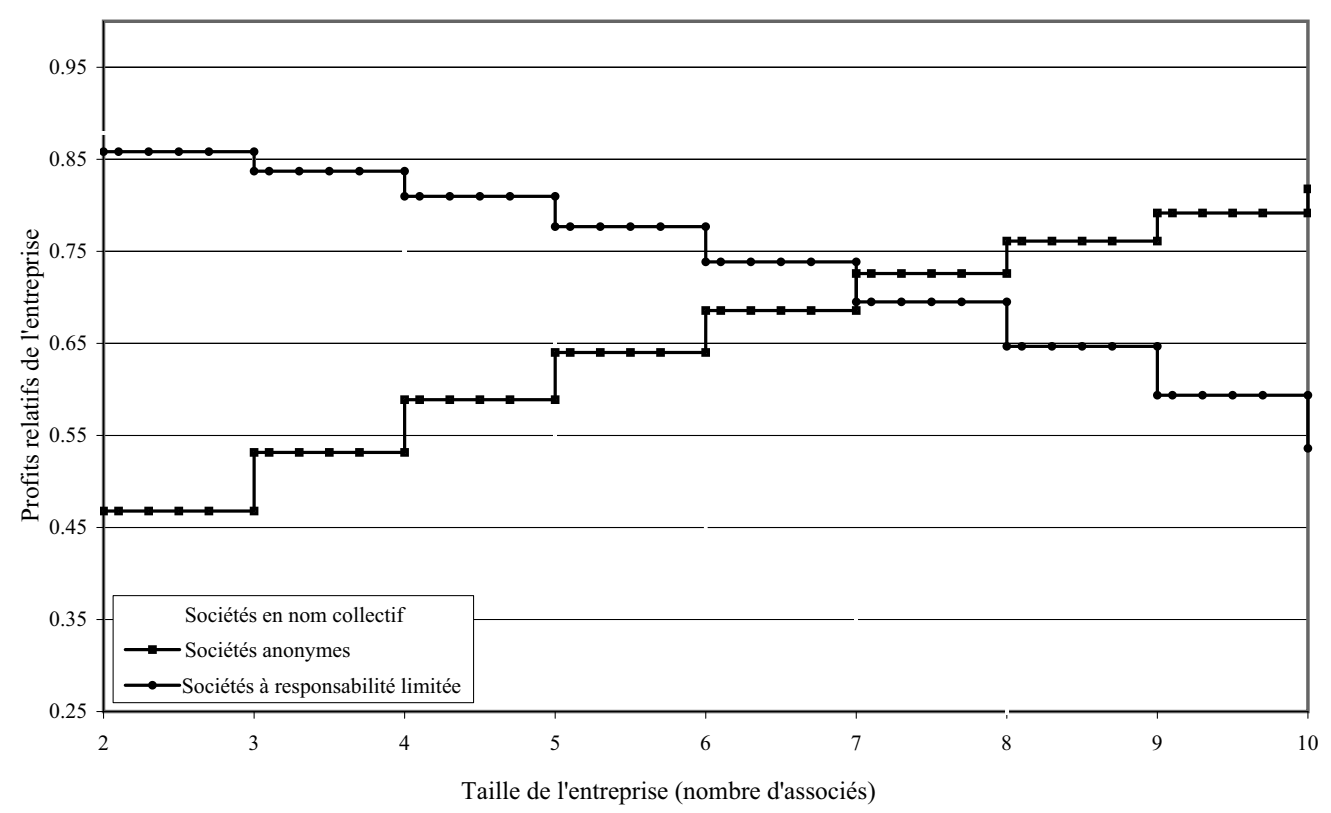

L'axe horizontal indique la taille de la société (nombre d'investisseurs) et l'axe vertical les bénéfices de l'entreprise (représentés comme une fractionde ses revenus si elle ne devait supporter aucun de ces coûts de transaction). La ligne représentant la proportion de ce profit maximum pour les SNC de différentes tailles est en baisse rapide parce que la probabilité de dissolution prématurée augmente rapidement avec le nombre d'associés. La ligne représentant la société anonyme est ascendante car cette dernière est plus avantageuse pour les grandes entreprises mais la forme de la ligne importe peu: elle pourrait même être déclinante, à condition que le déclin ne soit pas plus fort que celui des sociétés en nom collectif (possibilité peu réaliste car il existe peu de grandes entreprises organisées en SNC). Sur le diagramme, les lignes des SNC et des SA se croisent pour des entreprises comprenant moins de cinq investisseurs. Au-dessous de ce seuil, les sociétés seront en nom collectif parce que leurs bénéfices seront plus élevés, alors que celles ayant au moins cinq investisseurs seront des sociétés anonymes.

Imaginons maintenant qu'il existe un troisième choix, la SARL, représentée par la ligne en pente douce. Puisque les fondateurs de SARL peuvent réduire le risque de dissolution prématurée sans que le risque de l'oppression de la minorité devienne aussi grave que dans une SA, les avantages de cette forme apparaissent même dans une entreprise de plus petite taille (dans le diagramme, trois investisseurs). La SARL demeure la forme optimale d'organisation jusqu'à ce que l'entreprise soit d'une taille relativement grande (sept à huit investisseurs). En l'absence de SARL, les sociétés de trois à sept membres auraient choisi tantôt d'être des SNC, tantôt d'être des SA. En d'autres termes, la SARL «mange»des sociétés en nom collectif et des sociétés anonymes. 
L'analyse précédente suppose que toutes les formes d'organisation puissent être adoptées à peu de frais. C'était certainement le cas pour les SNC et les SARL; en revanche, il est possible que les sociétés anonymes aient été coûteuses au moment de leur création et tout au long de la vie de l'entreprise. Pour refléter ces coûts, décalons la ligne des sociétés anonymes vers la droite et rendons-la plus pentue (graphique 2). Avec ce scénario qui rend compte du cas allemand après 1884, la SARL est préférée à la société en nom collectif pour les entreprises de même taille que dans le scénario précédent, tandis qu'en son absence, les sociétés en nom collectif deviennent la forme d'organisation préférée pour les entreprises de moins de sept associés. Dans ce cas, les SARL supplantent davantage de sociétés en nom collectif que de sociétés anonymes. De façon générale, cependant, le rapport des SARL au nombre total des entreprises ne devrait guère changer entre ce scénario et le précédent qui suppose que la création de sociétés anonymes s'effectue à peu de frais.

\section{Graphique 2 - Impact d'une loi SARL quand la formation des SA est difficile}

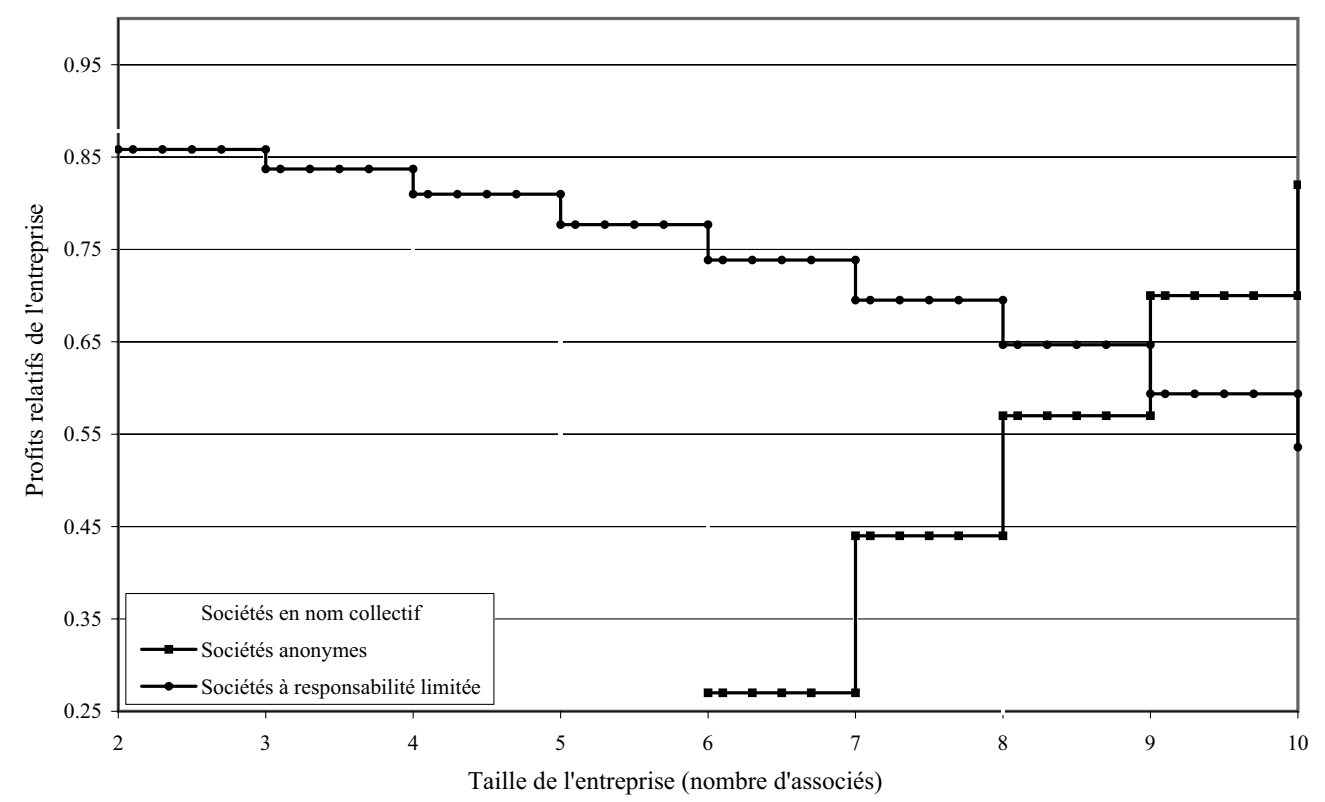

Les résultats restent proches si on déplace la ligne des sociétés en nom collectif vers le haut ou vers la droite. Un tel décalage peut se produire lorsqu'il existe des formes intermédiaires comme la société en commandite ou quand les sociétaires introduisent des clauses contractuelles restreignant leur capacité à sortir de l'entreprise. Soulignons surtout que la part des sociétés anonymes dans les sociétés à propriétaires multiples et la diffusion des SARL dépendent de l'ensemble des règles légales qui régissent les formes alternatives d'entreprises. Dans chacun des pays étudiés, l'alternative principale à la société anonyme avant l'avènement des SARL était la société en nom collectif. En France et en Allemagne, 
cependant, les sociétaires avaient d'autres options, ce qui a réduit l'attrait des sociétés anonymes et, dans une certaine mesure, a affecté la diffusion de la SARL. Néanmoins, comme nous le montrerons, l'analyse de base tient toujours.

\section{Des structures d'entreprise avant la SARL}

Au début du XIX ${ }^{\mathrm{e}}$ siècle en France et dans les trois autres pays, il fallait une autorisation spéciale du gouvernement pour former une société anonyme. Cependant, le Code de commerce de 1807 offrait aux commerçants ou aux entrepreneurs deux options en sus de la société en nom collectif. La société en commandite simple permettait à certains associés de limiter leur responsabilité à condition de ne pas jouer un rôle actif dans la gestion. On pouvait aussi organiser des commandites par actions, dans lesquelles les parts des commanditaires étaient cessibles. Dès les années 1830, il existait un marché pour les actions des sociétés en commandite qui offraient donc plusieurs des avantages des sociétés anonymes et étaient formées sans permission du gouvernement.

La loi allemande, influencée par les réformes françaises, offrait essentiellement les mêmes choix. Si les sociétaires ne voulaient pas former une association ordinaire (Offene Handelsgesellschaft ou $\mathrm{OHG}$ ), ils pouvaient se retrouver dans une commandite simple (Kommanditgesellschaft ou $\mathrm{KG}$ ) ou dans une commandite par actions (Kommanditgesellschaft auf Aktien ou KGaA). Après 1861, la plupart des États allemands ont adopté un code de commerce commun (l'Allgemeine Deutsche Handelsgesetzbuch ou ADHG), de sorte que les traits fondamentaux de ces sociétés sont devenus les mêmes dans toute l'Allemagne ${ }^{9}$.

La plus grande liberté contractuelle que les diverses formes de commandite ont donnée aux entrepreneurs français et allemands leur a permis de traiter un peu mieux le double risque de la dissolution prématurée et de l'oppression des minoritaires. Pour partie, en effet, les commandites étaient établies pour des durées spécifiques et les commanditaires ne pouvaient pas se retirer avant terme : le risque de dissolution prématurée était donc moindre que dans une société en nom collectif. En revanche, comme les associés passifs ne pouvaient pas participer à la gestion de l'entreprise, ils étaient sans doute plus souvent victimes des associés-gérants. Ce problème était cependant moins grave que dans une société anonyme parce que les associés passifs pouvaient se faire rembourser à la fin du contrat s'ils estimaient avoir été mal traités ${ }^{10}$.

9 - La première loi commerciale de l'empire fut votée en 1898, mais l'ADHG couvrait la plupart des aspects du droit des sociétés. Pour plus de détails, voir Timothy W. Guinnane et al., "Putting the corporation in its place ", working paper $\mathrm{n}^{\circ} 13109$, National Bureau of Economic Research, Inc., 2007.

10 - Philippe Merle, Droit commercial: sociétés commerciales, Paris, Dalloz, 1998; Michel DE JUGLART et Benjamin IPPOLITO, Les sociétés commerciales : cours de droit commercial, Paris, Montchrestien, 1999; Id., Protection des minoritaires : sociétés commerciales ne faisant pas appel public à l'épargne, Paris, Éd. Francis Lefebvre, 2001, p. 140. 
Au Royaume-Uni et aux États-Unis, les associés n'avaient pas accès à ces formes intermédiaires. Au Royaume-Uni, jusqu'en 1907 - soit longtemps après l'adoption de la loi libéralisant la société anonyme (corporation) -, il n'y a pas eu de loi autorisant les sociétés en commandite (limited ou sleeping partnership) et les tribunaux ont bloqué toute tentative d'en créer par contrat ${ }^{11}$. La situation était semblable aux États-Unis. Bien que, dans les années 1820 et 1830, la plupart des États aient autorisé les sociétés en commandite simple, les tribunaux ont interprété leurs statuts de façon si restrictive qu'elles n'ont jamais fourni une alternative aux sociétés en nom collectif ${ }^{12}$.

Le manque de solutions de rechange a peut-être induit ces deux pays à innover en matière de sociétés anonymes. Au Royaume-Uni, les chartes autorisant une société anonyme étaient des produits rares et chers jusqu'au deuxième tiers du XIX ${ }^{\mathrm{e}}$ siècle. Les parlementaires étaient alors sensibles aux pressions des entrepreneurs rivaux qui cherchaient à empêcher leur concurrent d'obtenir le statut de société anonyme. Puis le parlement a autorisé le Board of Trade à accorder des chartes plus librement et a permis la création par enregistrement de sociétés anonymes sans responsabilité limitée (en 1844), puis à responsabilité limitée (en 18551856). La loi de 1862 sur les sociétés, qui a consolidé ces acquis, a souligné la nature contractuelle des sociétés anonymes. Elle comprenait un modèle de statuts type (appelée Table A). Les sociétaires pouvaient adopter le modèle en entier, le rejeter tout à fait et rédiger leurs propres statuts, ou encore l'adopter en partie et remplacer certaines dispositions par d'autres là où ils le souhaitaient ${ }^{13}$.

Aux États-Unis, la constitution fédérale a laissé le droit des entreprises aux États. Dès 1809 et 1811, le Massachusetts et New York ont passé des lois permettant la création de sociétés anonymes par simple enregistrement afin de stimuler l'industrie locale à l'aube de la guerre de 1812. Pendant les quatre décennies suivantes, de telles lois se sont multipliées pour des secteurs de plus en plus variés de l'économie. Beaucoup d'États, particulièrement en Nouvelle-Angleterre, se sont aussi montrés très prodigues et ont accordé quantité de chartes par acte législatif spécial. Loin de ralentir les efforts pour démocratiser l'accès aux sociétés anonymes, cette politique a cependant plutôt stimulé l'adoption de lois permettant

11 - Les innovations telles que la société par actions non enregistrée pouvaient offrir plus de liquidités aux investisseurs, mais une société constituée sur cette base ne surmontait pas les défauts du nom collectif, puisque tel restait bien son statut au regard de la loi. Le trust était une autre possibilité, mais il n'a pas évolué suffisamment avant la loi sur les sociétés anonymes pour fournir une vraie option. L'acte de 1844 libéralisant les sociétés anonymes a aussi mis fin aux compagnies non enregistrées. Il a déclaré les sociétés par actions non inscrites illégales et interdit la formation des sociétés en nom collectif avec plus de 25 membres. Voir Ron HARRIS, Industrializing English law: Entrepreneurship and business organization, 1720-1844, Cambridge, Cambridge University Press, 2000.

12 - Naomi R. LamoreauX et Jean-Laurent RosenthaL, "Legal regime and contractual flexibility: A comparison of business's organizational choices in France and the United States during the era of industrialization ", American Law and Economics Review, 7-1, 2005, p. 28-61.

13 - R. HARris, Industrializing English law..., op. cit.; Bishop C. HunT, The development of the business corporation in England, Cambridge, Harvard University Press, 1936. 
leur création par enregistrement et, dès le début des années 1850, la plupart des États avaient choisi un processus administratif simplifié ${ }^{14}$.

En France, au contraire, jusqu'à la réforme de 1867, les chartes de sociétés anonymes ont été accordées avec parcimonie. Alors que, dans le Massachusetts, 2062 sociétés anonymes ont reçu une charte par acte législatif entre 1800 et 1862 , les gouvernements français successifs n'en ont autorisé que 642 entre 1800 et 1867. Après le Code de commerce français de 1807, la demande avait été faible du fait de la possibilité de créer des commandites par actions. En 1857, cependant, une crise financière donna lieu à des plaintes d'actionnaires minoritaires et de créanciers au sujet d'abus commis par les associés-gérants. Le gouvernement y répondit en rendant la création de commandites par actions plus difficile et onéreuse. Une fois la crise passée, cette mesure est cependant apparue malavisée et a conforté les efforts faits pour obtenir un régime de société anonyme plus libéral. La loi de 1863 a permis à des entreprises possédant moins de 20 millions de francs de capital d'être organisées comme des sociétés anonymes par simple enregistrement sans permission spéciale de l'État. Cependant, la nouvelle législation préservait les obligations de 1857 quant aux responsabilités des gérants et des membres des conseils d'administration et de surveillance. Elles étaient même alourdies dans la mesure où les membres du conseil d'administration et gérants se voyaient obligés de posséder au moins $5 \%$ des actions. L'acte de 1867 a supprimé la limite sur la capitalisation et soumis les sociétés anonymes et les commandites par actions aux mêmes règles libérales. En particulier, la responsabilité comptable des administrateurs a été réduite et les conditions minimales de propriété ont été éliminées ${ }^{15}$.

En Allemagne, l'ADHG ayant laissé la question des sociétés anonymes aux différents États, ceux-ci ont opté pour une création par simple enregistrement à des dates différentes. Les villes de la Hanse furent parmi les premières à lever les restrictions. La confédération allemande du Nord (menée par la Prusse) suivit en 1870 , et ses dispositions furent reprises par la loi impériale de $1871^{16}$. Deux facteurs ont joué un rôle dans la diffusion de la création simplifiée des sociétés anonymes. Les gouvernements ont appris qu'un rationnement des chartes n'empêchait pas la création de grandes entreprises, puisque les entrepreneurs pouvaient former des

14 - Voir Pauline MAIER, «The revolutionary origins of the American corporation », The William and Mary Quarterly, 50, 1993, p. 53-58; William C. KessLer, «A statistical study of the New York General Incorporation Act of 1811 », Journal of Political Economy, 48, 1940 , p. 877-882; James W. HuRST, Legitimacy of the business corporation in the law of the United States, 1780-1970, Charlottesville, University Press of Virginia, 1970, p. 13-57; Susan P. Hamill, «From special privilege to general utility: A continuation of Willard Hurst's study of corporations ", American University Law Review, 81, 1999, p. 81-180. 15 - Charles E. Freedeman, Joint-stock enterprise in France, 1807-1867: From privileged company to modern corporation, Chapel Hill, University of North Carolina Press, 1979, p. $132-135$.

16 - Norbert HoRn, «Aktienrechtliche Unternehmensorganisation in der Hochindustrialisierung (1860-1920)", in N. HORN et J. KOCKA (éd.), Recht und Entwicklung der Grossunternehmen im 19. und frühen 20. Jahrhundert: wirtschafts-, sozial- und rechtshistorische 
commandites par actions (KGaA) pour lever des capitaux sur le marché. Par exemple, la Disconto-Gesellschaft a pris la forme d'une commandite par actions pendant les années 1856-1867 alors qu'elle était la plus grande banque d'Allemagne ${ }^{17}$. De plus, avant 1871, moyennant contreparties fiscales ou autres, certains États avaient accordé des chartes de société anonyme à des entreprises légalement situées sur leur territoire, mais faisant la majeure partie de leurs affaires ailleurs dans le Zollverein. Les gouvernements ont donc appris là, à leurs dépens, qu'une politique restrictive n'empêchait pas des sociétés anonymes de fonctionner sur leur territoire mais menait seulement ces entreprises à recevoir leur charte d'un autre État allemand ${ }^{18}$.

\section{La lente diffusion des sociétés anonymes}

Au-delà de la chronologie de la législation sur les sociétés anonymes, si celles-ci étaient vraiment des structures aussi efficaces que l'on dit, on s'attendrait à ce que ces innovations législatives débouchent partout sur une augmentation massive de leur nombre. En France et en Allemagne, cependant, elles se sont avérées beaucoup moins populaires qu'aux États-Unis ou au Royaume-Uni. À la veille de la Première Guerre mondiale, on en comptait seulement 5000 en Allemagne et 13000 en France, contre 63000 au Royaume-Uni et plus de 250000 aux ÉtatsUnis ${ }^{19}$. En outre, même aux États-Unis et au Royaume-Uni, la majorité des entreprises à propriétaires multiples continua à être organisée en sociétés en nom collectif.

Le graphique 3 montre la répartition des nouvelles entreprises françaises par type légal en France de 1852 à $1978^{20}$. Le vote de la loi de 1867 est suivi par une augmentation subite du nombre de sociétés anonymes mais, excepté un bref pic à la fin des années 1870, elles n'ont jamais atteint plus de $10 \%$ des nouvelles sociétés avant la Première Guerre mondiale. Si les sociétés en nom collectif sont devenues relativement moins fréquentes pendant cette période, elles ont toujours représenté plus de $60 \%$ des nouveaux enregistrements et une partie de leur recul s'explique par un retour en faveur des commandites simples à la fin du XIX ${ }^{\mathrm{e}}$ siècle. Les commandites par actions, elles, ont subi un déclin continu, sans toutefois disparaître complètement en dépit du fait qu'elles ne constituaient qu'un mauvais substitut aux sociétés anonymes: Schneider est resté une commandite par action jusqu'aux années 1960, et Michelin en est toujours une aujourd'hui.

17 - Carsten Burhop, Die Kreditbanken in der Gründerzeit, Stuttgart, Franz Steiner Verlag, 2004, p. 84

18 - Rondo Cameron, «The founding of the Bank of Darmstadt », Explorations in Entrepreneurial History, 8-3, 1956, p. 112-130.

19- Charles E. Freedeman, The triumph of corporate capitalism in France 1867-1914, Rochester, University of Rochester Press, 1993, p. 21; Susan B. CARTER et al., Historical statistics of the United States: Earliest times to the present, New York, Cambridge University Press, 2006, séries Ch 1-18.

20 - Compte général de l'administration de la Justice civile et commerciale en France et Annuaire statistique de la France. Ministère de l'agriculture et du commerce, Service de la statistique générale de France. 


\section{Graphique 3 - Distribution des nouvelles sociétés par type juridique en France,} 1852-1978

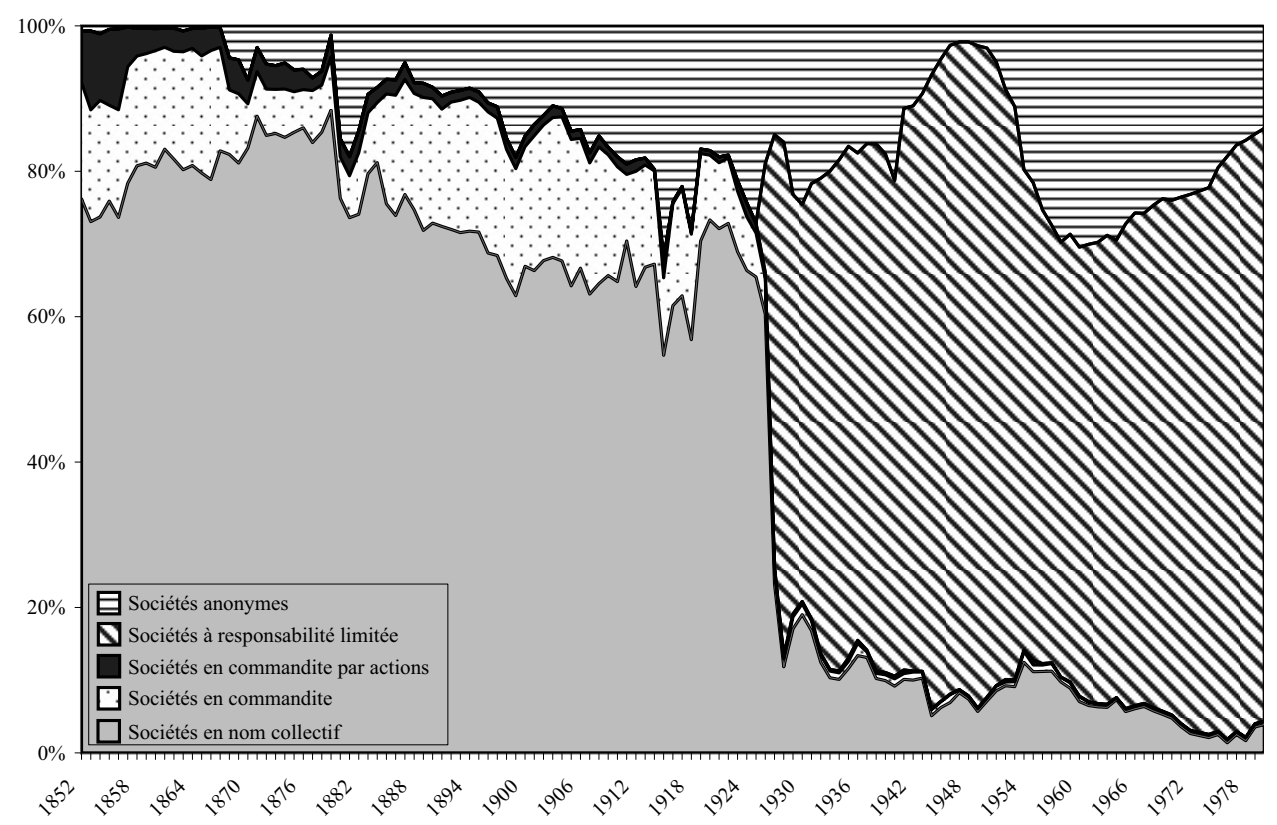

Sources: Compte général de la Justice et Annuaire statistique de la France.

Les données pour 1914-1918 ont été interpolées à partir des totaux pour Paris dépouillés aux archives du tribunal de commerce de Paris.

Le faible nombre de sociétés anonymes créées en France après 1867 ne semble pas découler de coûts de création élevés ou de règles d'administration contraignantes. Toutes les sociétés commerciales étaient soumises à la même fiscalité et la publicité supplémentaire à laquelle les sociétés anonymes étaient tenues ne constituait pas une charge bien lourde dès lors que les exigences rigoureuses de la loi de 1857 avaient été supprimées. Après 1907, les sociétés faisant un appel public à l'épargne ont dû publier une notice d'information, déposer une copie de leurs statuts, la liste de leurs actionnaires initiaux et le compte rendu de leurs premières assemblées générales. Mais, à part le prospectus, les autres sociétés étaient soumises aux mêmes formalités et les frais d'enregistrement étaient essentiellement les mêmes pour toutes. La plupart des sociétés anonymes se constituaient par acte notarié (un coût représentant $1 \%$ du capital), bien que ce ne fût pas là une obligation légale ${ }^{21}$. Il est donc probable que leur diffusion limitée s'explique par l'existence des commandites qui offraient une certaine protection contre la dissolution prématurée sans faire courir aux minorités autant de risques d'oppression. 
Le graphique 4 détaille les formes d'organisation choisies par les sociétés nouvelles enregistrées en Prusse de 1867 à $1932^{22}$. Le vote de la loi sur les sociétés anonymes a été suivi par un bref pic de popularité de celles-ci, car les entrepreneurs prussiens ont rapidement tiré profit de leur nouvelle liberté ${ }^{23}$. Comme en France, cependant, la société anonyme n'a pas remplacé les sociétés en nom collectif qui ont continué à dominer. Pour chaque nouvelle société anonyme, il y avait au moins 16 sociétés en nom collectif créées en 1882 et 41 en 1892. Les commandites sont également demeurées une forme d'organisation très pratiquée, et quand une société en nom collectif changeait de forme légale, la nouvelle entité était le plus souvent une commandite.

Beaucoup de sociétés anonymes furent créées pendant le «Gründerboom » de 1871-1873, quand le rapide paiement de l'indemnité imposée à la France après 1870 produisit une bulle spéculative sur le marché boursier ${ }^{24}$. Bien des entreprises nouvelles furent financées dans l'illusion de gains rapides ou par des fraudes audacieuses, et l'éclatement de la bulle en élimina un grand nombre. Sur les 1005 sociétés anonymes formées entre 1867 et 1873, 123 étaient en liquidation en septembre 1874 et 37 en faillite ${ }^{25}$.

L'éclatement de la bulle eut aussi pour conséquence l'adoption de la loi de 1884 visant à augmenter le pouvoir des actionnaires et à réduire certains abus dans la formation de nouvelles entreprises. Le rôle du conseil de surveillance (Aufsichtsrat) fut renforcé et les sociétés anonymes durent fournir des bilans plus détaillés ${ }^{26}$. D'autre part, la valeur minimum des actions est passée de 100 à

22 - Les statistiques sur les formes d'entreprise couvrant toute l'Allemagne commencent en 1887. Avant, seule la Prusse a publié des informations détaillées sur ce sujet. Nos données détaillées sur les nouvelles sociétés viennent du Königlich Preussischer StaatsAnzeiger avant 1871 et du Reichsanzeiger après cette date. Cette source hebdomadaire publie les notices légales qui incluent les résumés des statuts des entreprises commerciales. Nous avons transcrit toutes les informations concernant les formations, dissolutions et transformations de tous les types d'entreprises pour les mois de janvier des années se terminant par le chiffre 2 ou le chiffre 7 comme 1867, 1882, 1887, 1892 jusqu'en 1932. Un dépouillement complet sur deux années, en 1891 et 1892, montre que le rythme de création des divers types de société n'est guère sensible à la saisonnalité. La Prusse comprenait la majorité de la population du territoire et de l'activité économique du Reich formé en 1871. Des 4712 sociétés anonymes existant en Allemagne en 1912, 2619 se trouvaient en Prusse, 384 en Bavière, 454 en Saxe et 140 dans le Wurtemberg. La part de la Prusse dans le nombre des sociétés anonymes est légèrement moindre que son poids dans la population allemande; elle est aussi plus faible que sa part du PNB. 23 - Pour un examen détaillé des sociétés formées en Prusse après 1871, voir Ernst ENGEL, «Die erwerbsthätigen juristischen Personen im preussichen Staate. Insbesondere die Actiengesellschaften », Zeitschrift des Königlichen Preussischen Statistischen Bureaus, $15,1875$.

24 - C. Burhop, Die Kreditbanken..., op. cit., p. 25. Il donne un index de marché boursier $(1870=100)$ qui plafonne à 186 en novembre 1872 et tombe au-dessous de 100 en mai 1875 .

25 - Eduard WAGON, Die finanzielle Entwicklung deutscher Aktiengesellschaften von 1870-1900 und die Gesellschaften mit beschränkter Haftung im Jahre 1900, Iéna, Gustav Fisher Verlag, 1903, p. 3.

26 - Il n'y avait pas d'audit externe avant 1931; le conseil de surveillance était censé être un auditeur interne. 


\section{Graphique 4 - Distribution des nouvelles sociétés par type juridique en Prusse, 1867-1932}

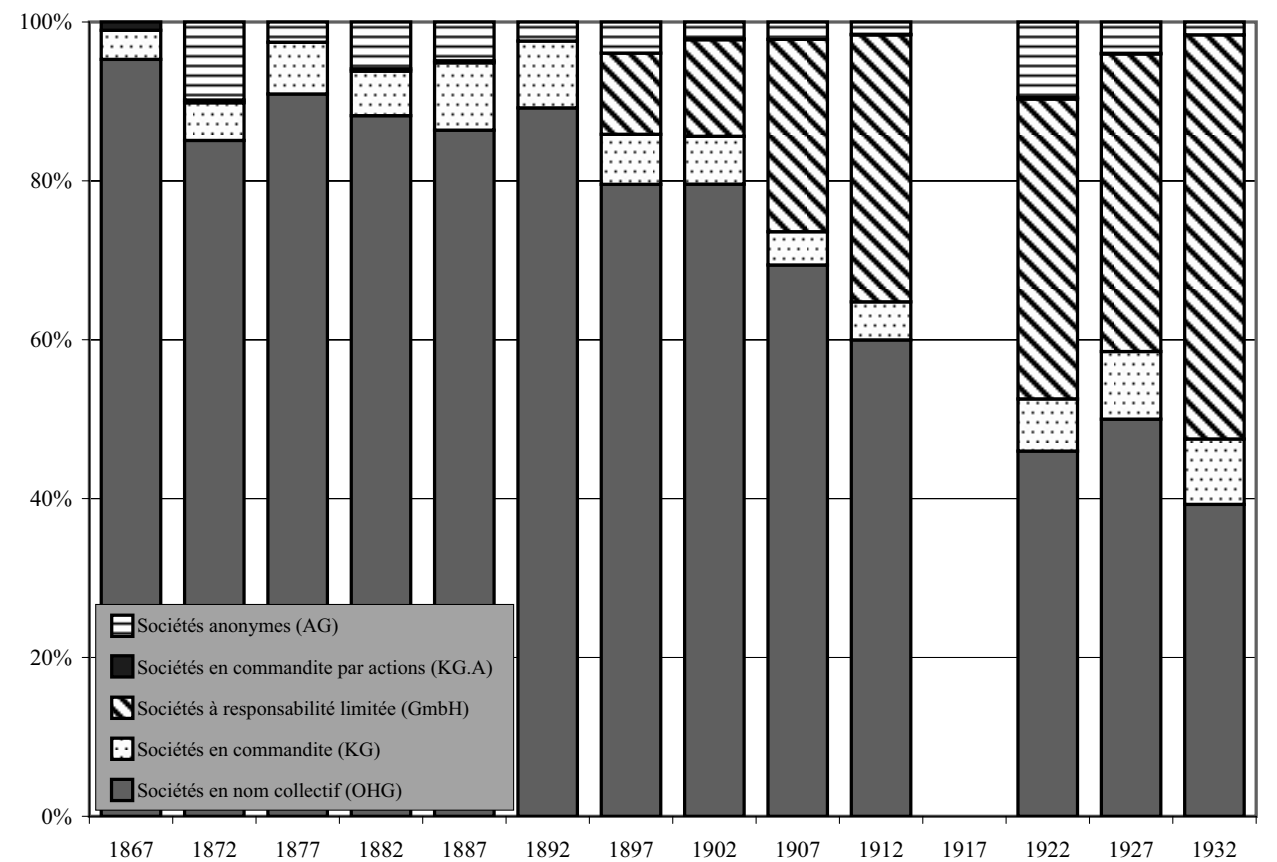

Sources: Les totaux par type ont été extraits du Königlich preussischer StaatsAnzeiger (jusqu'en 1871) puis du Deutscher Reichsanzeiger und preussischer Staatsanzeiger. Nous avons énuméré toutes les entreprises qui ont publié leur création ou transformation en janvier de chaque année.

1000 marks; toutes les parts devaient obligatoirement être souscrites avant que l'entreprise puisse agir et les sociétés qui choisissaient de devenir des sociétés anonymes ne pouvaient être cotées en bourse durant la première année suivant leur réorganisation ${ }^{27}$.

La loi de 1884 a sans doute rendu la société anonyme moins attrayante et le nombre d'enregistrements est resté en dessous de 400 par an pour le reste du siècle ${ }^{28}$. Quelques entreprises ont adopté d'autres formes. Jürgen Kocka et Hannes Siegrist notent que 15 des 100 plus grandes entreprises industrielles allemandes

27 - Gesetz, betreffend die Kommanditgesellschaften auf Aktien und die Aktiengesellschaften, vom 18. Juli 1884, commenté par P. Kayser, Berlin, H. W. Müller, 1884.

28 - Comme Timothy W. Guinnane l'a montré, ces réformes ont renforcé le rôle des grandes banques dans la formation des sociétés: Timothy W. Guinnane, « Delegated monitors, large and small: Germany's banking system, 1800-1914 », Journal of Economic Literature, 40, 2002, p. 104-105. Caroline Fohlin met aussi l'accent sur la réforme de 1884, mais s'intéresse plus aux banques: Caroline FoHLIN, "Regulation, taxation, and the development of the German universal banking system, 1884-1913 », European Review of Economic History, 6, 2002, p. 221-254. 
étaient Personengesellschaften (sociétés de personnes ou firmes uni-propriétaires) en 1887 et 7 seulement en $1907^{29}$. Aujourd'hui encore, certaines des plus grosses sociétés allemandes sont organisées comme des sociétés en nom collectif d'un type ou d'un autre. Ainsi, la compagnie qui regroupe la direction allemande de Merck est une commandite par actions (KGaA) ${ }^{30}$.

Les entrepreneurs britanniques et américains n'avaient que deux options, la société anonyme ou la société en nom collectif. Il n'est donc pas étonnant qu'ils aient adopté la première plus rapidement que les Français ou les Allemands. Pourtant, plusieurs décennies après le vote des lois sur les sociétés anonymes, la majorité des sociétés ont continué à être en nom collectif. Aux États-Unis, jusqu'en 1914, la concurrence entre États pour établir des chartes de sociétés anonymes a réduit à peu de chose la plupart des restrictions mises à la création par enregistrement et a contribué à réduire ses coûts ${ }^{31}$. Les créations de sociétés ont été multipliées par vingt entre 1870 (date à laquelle déjà plusieurs milliers étaient enregistrées chaque année) et $1925^{32}$. Néanmoins, les données du recensement de l'industrie indiquent qu'en 1900, plus d'un demi-siècle après les lois sur les sociétés anonymes, les sociétés en nom collectif constituaient toujours plus de $60 \%$ des entreprises multipropriétaires. Dans les vingt ans qui ont suivi, marqués par une forte augmentation de la taille des entreprises, la proportion des industriels choisissant cette forme de société est tombée à environ $40 \%$, mais cette chute s'est ensuite arrêtée jusqu'après la Deuxième Guerre mondiale. Selon des données du Trésor (IRS), en 1947, les sociétés en nom collectif représentaient encore au moins $40 \%$ des entreprises multi-propriétaires dans l'industrie, et $60 \%$ dans l'économie dans son ensemble (voir le tableau 1, p. 100). C'est seulement au lendemain de la guerre, quand la société anonyme a bénéficié d'avantages fiscaux substantiels, que la proportion de sociétés en nom collectif a amorcé un déclin rapide ${ }^{33}$.

Bien qu'il n'y ait pas de sources semblables pour le Royaume-Uni, les évaluations disponibles montrent que les sociétés en nom collectif y ont tout aussi longtemps gardé une place importante. En 1872, la commission sur les SNC a débattu d'une éventuelle taxation sur l'enregistrement des sociétés commerciales. Une

29 - Jürgen Kocka et Hannes SIEgRIST, «Die hundert größten deutschen Industrieunternehmen im späten 19. und frühen 20. Jahrhundert», in N. HorN et J. KockA (éd.), Recht und Entwicklung..., op. cit., p. 80-81, tabl. 1 et 2.

30 - Le gérant est une association ordinaire (OHG) détenue par la famille Merck ; Henkel KGaA et la banque Oppenheim sont d'autres entreprises à propriétaires multiples importantes en Allemagne.

31 - Arthur K. KuHN, A comparative study of the law of corporations with particular reference to the protection of creditors and shareholders, New York, Columbia University, 1912 ; Christopher GRANDY, «The economics of multiple governments: New Jersey corporate chartermongering, 1875-1929», Ph. D., University of California, Berkeley, 1987.

32 - George H. Evans Jr., Business incorporation in the United States, 1800-1943, New York, National Bureau of Economic Research, 1948, p. 34.

33 - N. R. Lamoreaux et J.-L. Rosenthal, "Contractual tradeoffs... », op. cit.; S. B. Carter et al., Historical statistics of the United States..., op. cit.; W. Elliot BrownLEE, Federal taxation in America: A short history, Washington/Cambridge/New York, Woodrow Wilson Center Press/Cambridge University Press, 1996, p. 89-129. 
évaluation plutôt conservatrice de 1885 a estimé le nombre «de sociétés en nom collectif importantes " à environ 100000 et a calculé que les sociétés anonymes ne représentaient pas plus de 5 à $10 \%$ «des firmes importantes», entreprises individuelles exclues. Les quelques autres évaluations disponibles indiquent que le nombre de grandes entreprises organisées en sociétés anonymes dans la sidérurgie et les transports maritimes a augmenté pendant les deux dernières décennies du XIX ${ }^{\mathrm{e}}$ siècle, il en va de même dans l'industrie du coton au début du XXe siècle. Même dans les secteurs dominés par de grandes sociétés, il a donc fallu attendre plus de cinquante ans après la libéralisation des sociétés anonymes pour que ces dernières deviennent majoritaires. On connaît moins bien les formes d'organisation adoptées par les PME: la plupart de ces entreprises devaient être des sociétés en nom collectif quand la SARL fut introduite au Royaume-Uni ${ }^{34}$.

L'histoire de ces quatre pays ne vient donc pas conforter l'idée reçue selon laquelle la société anonyme est la forme supérieure d'organisation. Relativement peu de sociétés anonymes ont été formées en France et en Allemagne et, même aux États-Unis et au Royaume-Uni, la majorité des entreprises multi-propriétaires a continué à s'organiser en sociétés de personnes. C'est seulement en Allemagne, après 1884 , que l'impopularité des sociétés anonymes peut être mise sur le compte de lois restrictives. De façon générale, les entrepreneurs les ont évitées parce que d'autres formes d'organisation répondaient mieux à leurs besoins. Au RoyaumeUni et aux États-Unis, les entrepreneurs désireux éviter l'oppression des minoritaires se sont organisés en sociétés en nom collectif, quitte à prendre le risque d'une dissolution prématurée. En France et en Allemagne, ils ont privilégié la société en commandite, mieux à même de les protéger contre une dissolution rapide sans les menacer autant qu'avec une SA du risque d'oppression.

\section{La $\mathrm{GmbH}$ et sa diffusion en Allemagne}

La législation des SARL (Gesellschaft mit beschränkter Haftung ou GmbH) en Allemagne fut une conséquence directe des mesures prises en 1884. Celles-ci avaient rendu la formation de nouvelles sociétés anonymes si coûteuse que beaucoup d'observateurs de l'époque ont estimé qu'elles avaient favorisé la concentration de la puissance économique en Allemagne. Les revendications ont alors porté sur une réforme de la société anonyme et sur la mise en place d'une nouvelle forme de société. Dès 1888 le ministre prussien du commerce demanda à l'association des commerçants allemands d'en discuter, et en 1892 le Reichstag vota la loi créant les sociétés à responsabilité limitée $(\mathrm{GmbH})$. La forme en fut légèrement modifiée en 1898 quand le code commercial (Handelsgesetzbuch ou HBG) fut instauré ${ }^{35}$.

\footnotetext{
34 - James B. JefFerys, Business organisation in Great Britain, 1856-1914, New York, Arno Press, 1977.

35 - Werner SchuberT, «Die Gesellschaft mit beschränkter Haftung. Eine neue juris- 
Deux personnes ou plus pouvaient alors créer une société à responsabilité limitée: il leur suffisait d'inscrire dans le registre de commerce local des statuts conformes à la législation (Gesellschaftsvertrag). Une société à responsabilité limitée devait posséder au moins 20000 marks de capital souscrit (Stammkapital) ${ }^{36}$. Ce capital pouvait être divisé en parts d'au moins 500 marks. Différence importante avec une société anonyme : dans une $\mathrm{GmbH}$ il fallait un acte notarié pour transférer des parts et celles-ci ne pouvaient pas être cotées en bourse. Mais ce coût de transfert plus élevé mis à part, la loi imposait peu de contraintes. Par exemple, l'existence d'un conseil de surveillance n'était pas obligatoire. Une GmbH pouvait ou non avoir un ou plusieurs gérants (Gesellschaftsführer) choisis parmi les propriétaires de parts (que nous appellerons dorénavant actionnaires). Ces gérants représentant légalement la société, leur nom devait être inscrit au registre du commerce mais, en droit, les actionnaires pouvaient les limoger à tout moment ${ }^{37}$.

Bien que la loi ait stipulé quelques règles type permettant aux statuts d'être brefs et simples, la plupart des questions d'organisation importantes étaient laissées aux propriétaires de l'entreprise. Une société à responsabilité limitée pouvait être formée pour une période déterminée ou illimitée; dans les deux cas, les investisseurs étaient protégés de la menace de dissolution prématurée par une règle qui exigeait l'accord des détenteurs d'au moins $3 / 4$ de la valeur des parts pour liquider la société. Dans les statuts types, cependant, cette protection impliquait un plus grand risque d'oppression des minoritaires, comme dans une société anonyme. L'actionnaire recevait une voix pour 100 marks de capital investi, et la majorité simple suffisait pour choisir les gérants et prendre la plupart des autres décisions. Cela permettait donc aux propriétaires représentant $51 \%$ du capital d'imposer leur volonté aux minoritaires. Ce n'était là cependant que les dispositions type. Les organisateurs pouvaient se mettre d'accord sur d'autres règles acceptant, s'ils le voulaient, plus de risque de dissolution prématurée contre moins de danger d'oppression de minorité.

Revenons au graphique 4 qui quantifie les sociétés nouvelles enregistrées en Prusse tous les cinq ans à partir de 1867 : il est clair que le succès des sociétés à responsabilité limitée s'est affirmé lentement pendant la première décennie. À partir de 1912, cependant, environ un tiers des sociétés nouvelles a adopté cette forme, et après 1932 la moitié d'entre elles. Comme on pouvait s'y attendre, l'arrivée de la $\mathrm{GmbH}$ a eu peu d'effet sur les enregistrements de sociétés anonymes. Avant comme après 1892, celles-ci étaient extrêmement rares; entre 1872 et 1912, leur nombre varie peu: il ne s'en est formé chaque année qu'une vingtaine, en dépit de la croissance économique considérable de l'Allemagne pendant cette

36 - En 1892, 20000 marks équivalaient à environ 1000 \&, 4860 \$ et $20000 \mathrm{~F}$. C'était donc une somme importante (le PNB allemand par tête en 1892 était de 470 marks): Walther G. Hoffmann et al., Das Wachstum der deutschen Wirtschaft seit der Mitte des 19. Jahrhunderts, Berlin/Heidelberg/New York, Springer, 1965, p. 248, tabl. 1.

37 - Max Hachendurg, Staub's Kommentar zum Gesetz, betreffend die Gesellschaften mit beschränkter Haftung, Berlin, Gettentag, [1903] 1913, p. 441-442. Pour une description plus complète de la forme GmbH, voir T. W. Guinnane et al., «Putting the corporation in its place", op. cit. 
période. En raison du coût élevé de leur création, seules les entreprises attirées par les avantages spécifiques de ce type de société adoptaient cette forme ${ }^{38}$.

La société à responsabilité limitée n'a pas nui aux commandites qui ont continué à représenter environ $8 \%$ des entreprises. Comme le prévoit notre modèle, son apparition a principalement affecté les sociétés en nom collectif dont certains propriétaires furent attirés par la responsabilité limitée et par la transformation du capital en actions sans pour autant supporter les coûts d'une société anonyme ou les risques accrus d'oppression des minoritaires inhérents aux sociétés anonymes et aux commandites simples. En Prusse, la part des sociétés en nom collectif diminua de près de $90 \%$ en 1892 (juste avant l'arrivée des sociétés à responsabilité limitée) à moins de $40 \%$ quarante ans plus tard. On peut supposer que ce déclin aurait été encore plus grand si le capital minimum des sociétés à responsabilité limitée n'avait pas été si élevé. En 1913, un tiers des sociétés à responsabilité limitée n'avaient pour capital souscrit que les 20000 marks exigés comme minimum légal ${ }^{39}$.

\section{La private limited liability company au Royaume-Uni}

L'histoire de la SARL (Private limited liability company) au Royaume-Uni est très semblable à celle observée en Allemagne. Suite à des scandales où des promoteurs de sociétés avaient escroqué les investisseurs, le parlement adopta en 1900 une loi qui soumettait les appels publics à l'épargne à de nouvelles règles. Chaque compagnie émettant des actions devait désormais éditer un prospectus donnant aux investisseurs des informations détaillées sur l'entreprise. Elle limitait la capacité des sociétés à assigner des parts aux organisateurs ou à des proches qui ne les auraient pas payées comptant, requérait la publicité des hypothèques et des dettes comme moyen de protéger les créanciers sans garantie, et exigeait la diffusion de bilans certifiés lors des réunions annuelles d'actionnaires. Enfin, la loi rendait les administrateurs personnellement responsables en cas de non-respect de ces dispositions ${ }^{40}$.

Le but de la loi anglaise était de protéger les investisseurs des compagnies cotées mais elle a augmenté le coût des sociétés anonymes, que celles-ci soient ou non ouvertes au public. Comme la loi de 1884 en Allemagne, celle de 1900 au Royaume-Uni a ainsi réduit l'attrait de la société anonyme, particulièrement pour les PME : le nombre de compagnies enregistrées a chuté de 5082 en 1897 à 3725 en $1904^{41}$. Cette loi a également soulevé dans le monde des affaires des protestations

38 - Il n'est pas étonnant que peu de sociétés anonymes se soient converties en GmbH. Une société qui avait payé les coûts de création d'une SA n'avait probablement pas beaucoup de raisons d'abandonner cette forme.

39 - Avec le temps, la proportion des GmbH les plus petites s'est accrue. En 1904, $40 \%$ de toutes les GmbH en Prusse avaient un capital de 20000 à 50000 marks; en 1913, $58 \%$ : Statistisches Jahrbuch für den Preussischen Staat, Berlin, 1915, vol. 12, tabl. VII.B1, p. 221 et tabl. VII.B2, p. 222. 
grandissantes auxquelles le parlement répondit en 1905 par la nomination du comité Loreburn-Warmington dont les recommandations permirent en 1907 d'amender la loi en créant la société à responsabilité limitée privée (PLLC) ${ }^{42}$. Soulignons que le comité Loreburn-Warmington ne fit aucune référence dans son rapport à la $\mathrm{GmbH}$ comme une forme utile ou même comme modèle à éviter. Il prit une approche différente et recommanda des dispositifs spécifiquement britanniques ${ }^{43}$.

La loi de 1907 a créé une distinction entre entreprises publiques et privées, soumettant les premières à des règles plus strictes et à une publicité plus grande que les secondes. La section 37 de la loi indique qu'une entreprise privée anonyme «est une compagnie qui par ses articles» limite le droit de transférer ses parts, limite le nombre de ses actionnaires à cinquante, et s'interdit d'inviter le public à souscrire des parts ou obligations de la compagnie ${ }^{44}$. Alors qu'en Allemagne une compagnie devenait privée en vertu d'une loi qui la rendait différente d'une société anonyme, au Royaume-Uni une compagnie devenait privée en incluant dans ses statuts les dispositions ci-dessus, mais elle restait une société anonyme.

Les restrictions mises à la cessibilité des parts ont le plus souvent exigé soit le consentement du conseil ou d'autres actionnaires, soit que les parts soient offertes à d'autres membres de la société avant de pouvoir être transférées à des tiers. Bien que de telles dispositions aient augmenté la vulnérabilité des actionnaires minoritaires, les entrepreneurs pouvaient adopter des statuts qui corrigeaient ce déséquilibre. Les actionnaires minoritaires pouvaient ainsi être protégés en augmentant leur droit de vote ou en exigeant des majorités plus élevées pour certaines questions, même si la loi ne le stipulait pas. Toutefois, les amendements aux statuts ou une dissolution anticipée de la compagnie ne pouvaient être adoptés sans une majorité de $75 \%$. Une SARL pouvait être dissoute par jugement, mais seulement dans certains cas comme l'incapacité de payer ses dettes ou sur la constatation qu'il était «juste et équitable» de liquider l'entreprise ${ }^{45}$.

Le graphique 5 montre la proportion de nouvelles compagnies organisées en sociétés anonymes (publiques) comparées à celles qui l'ont été en SARL (PLLC). La SARL a connu une popularité énorme et quasi immédiate. En effet, si 4102 entreprises ont été enregistrées en tant que sociétés anonymes de 1902 à 1906, la chute fut ensuite spectaculaire. En revanche, les nouvelles SARL se sont multipliées: la moyenne annuelle est passée de 4853 entre 1907 et 1912, juste après la loi, à 7936 entre 1922 et 1926, puis 12350 entre 1932 et 1936. Après 1922, elles constituaient au moins $93 \%$ des sociétés par actions créées ${ }^{46}$. Bien que nos données ne permettent pas de comparer la taille moyenne des SARL à celle des sociétés anonymes,

42 - National Archives of the United Kingdom, Companies Act 1907 (7 Edw. 7 c. 50). 43 - Parliamentary Papers, 1906, vol. XLIV (Cd. 3052).

44 - National Archives of the United Kingdom, Companies Act 1907, section 37, private companies.

45 - National Archives of the United Kingdom, Companies (Consolidation) Act 1908 (8 Edw. 7 Ch. 69), section 129.

46 - UK Board of Trade, General annual report under the Companies (Winding-up) Act of 1890, Londres, 1900-1921; UK Board of Trade, General annual report under the Companies (Winding-up) Act of 1890, Londres, 1922-2000. 
notons que la modification de la loi a été accompagnée d'une augmentation du nombre de petites entreprises s'enregistrant comme sociétés. En 1901, 34,7\% des sociétés récemment formées avaient un capital social de $5000 £$ ou moins. Dès 1908 , ce pourcentage passe à 47,4\% pour atteindre $76,2 \%$ en 1936 . La corrélation entre l'augmentation du nombre de SARL et l'élévation du nombre de compagnies avec moins de $5000 £$ de capital suggère que beaucoup de petites entreprises ont choisi la SARL.

\section{Graphique 5 - Part des SARL dans les enregistrements de sociétés de capitaux au Royaume-Uni}

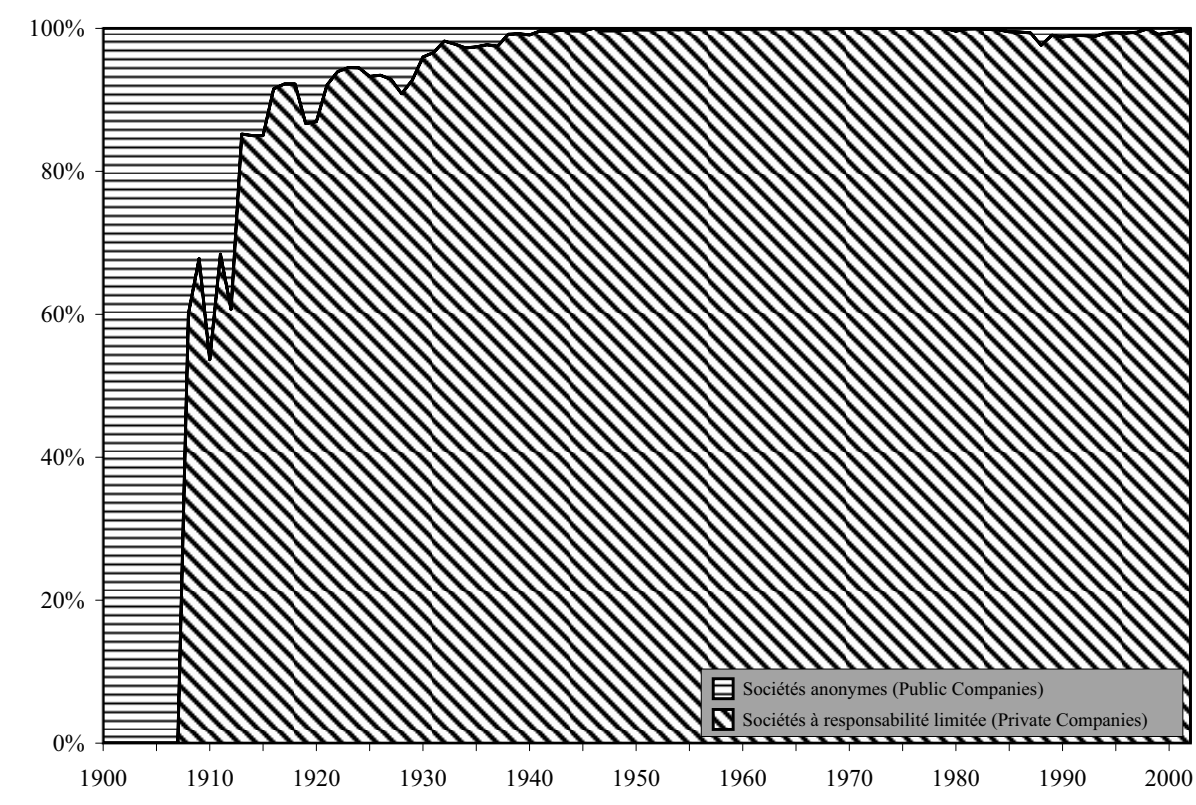

Source: UK Board of Trade, General annual report under the Companies (Winding-up) Act of 1890, Londres, 1900-1921; UK Board of Trade, General annual report under the Companies (Winding-up) Act of 1890, Londres, 1922-2000.

\section{La SARL en France}

En dépit de propositions récurrentes visant à réformer la loi de 1867 sur les sociétés anonymes, peu fut fait entre 1867 et 1925. C'est la fin de la Première Guerre Mondiale et le retour de l'Alsace et de la Lorraine qui poussèrent au changement. Les entrepreneurs alsaciens et lorrains avaient pu s'organiser en GmbH depuis 1892 et, en 1919, au moins 400 de ces sociétés existaient en Alsace-Lorraine. Leurs propriétaires étaient peu désireux de les convertir en sociétés en nom collectif, en commandites ou en sociétés anonymes ${ }^{47}$. Ils ont au contraire fait pression pour la légalisation 
des GmbH. En 1919, une proposition de loi visant à permettre la création d'entreprises sur un modèle proche des $\mathrm{GmbH}$ fut présentée à l'Assemblée, mais elle provoqua un tollé et fut retirée presque immédiatement: après quatre ans dans les tranchées, les vainqueurs ne voulaient pas imiter les perdants. Malgré la déception provoquée en Alsace-Lorraine, cet échec galvanisa les partisans d'une réforme et un nouveau projet de loi portant création de la SARL fut présenté en $1921^{48}$. Pour des raisons obscures, il ne fut approuvé qu'en 1925. Le type de SARL retenu était cependant très différent de celui de l'Allemagne ou du Royaume-Uni ${ }^{49}$.

Selon cette loi, pour former une SARL, les statuts de la société devaient être enregistrés au tribunal de commerce. Comme en Allemagne, la loi vise à réduire les coûts de publicité et de formation et, bien que les sociétés devaient être enregistrées, leurs statuts pouvaient être élaborés sous seing privé, évitant ainsi les frais de notaire. Comme pour la $\mathrm{GmbH}$, il fallait un capital minimum, fixé à 25000 francs, mais étant donné la valeur du franc, cette contrainte était faible. Le capital devait être divisé en parts de 100 francs ou plus ${ }^{50}$.

À la différence d'une société en nom collectif, une SARL était une société par actions et, comme telle, n'était pas dissoute par la mort d'un associé: les parts étaient transmises aux héritiers. Elle n'avait pas non plus à être dissoute dès lors qu'un associé était pris du désir de s'en retirer. Étaient ainsi résolues deux difficultés qui menaçaient sans cesse la pérennité des sociétés en nom collectif. Comme pour la GmbH et la PLLC, les parts de SARL ne pouvaient être cotées en bourse et n'étaient pas librement cessibles, mais la loi française est allée plus loin en soumettant les ventes de parts à l'aval des propriétaires de 3/4 des actions. À la différence du cas allemand, une vente approuvée pouvait être menée à bonne fin sans recourir à un notaire ${ }^{51}$.

En apparence, les actionnaires minoritaires d'une SARL risquaient d'être moins protégés que leurs équivalents dans les sociétés allemandes ou britanniques, parce qu'elle devait strictement respecter la règle : une part, une voix. En revanche, les gérants étant nommés dans les statuts, ils étaient inamovibles sauf par voie judiciaire et cette capacité de nommer ou non les gérants ajoutait une marge de souplesse. Considérons le cas d'une SARL sans actionnaire dominant. Selon la façon dont les statuts étaient rédigés, la société pouvait soit se structurer comme une société anonyme et décider que le gérant pouvait être renvoyé par un vote, soit

48 - Documents parlementaires. Chambre des députés, 1925-1966; Georges RIPERT, Traité élémentaire de droit commercial, Paris, Librairie Générale de Droit et de Jurisprudence, 1967-1970.

49 - Pour les projets, voir Documents parlementaires, Chambre des députés, 3348, novembre 1921; G. RIPERT, Traité élémentaire de droit commercial, op. cit., vol. 1, p. 476-478. Voir aussi Documents parlementaires, Chambre des députés, annexe 712, session du 16 décembre 1924, p. 691-699.

50 - Dans les années 1920, 25000 francs correspondaient à cinq fois le PNB par tête. "Loi du 7 mars 1925 ", Code de commerce, suivi des lois commerciales et industrielles, avec annotations d'après la doctrine et la jurisprudence et renvois aux publications Dalloz, Paris, Libraire Dalloz, 1967, p. 800-806.

51 - Pour plus d'informations sur ces dispositions et pour les changements de la loi au cours du $\mathrm{XX}^{\mathrm{e}}$ siècle, voir T. W. GuinNaNe et al., "Putting the corporation in its place ", op. cit. 
comme une commandite simple et dans ce cas le directeur ne pourrait pas être remplacé sans dissoudre la société. Si tous les membres de la société possédaient le même nombre de parts, une SARL pouvait même être structurée comme une société en nom collectif dans laquelle tous les membres avaient les mêmes pouvoirs.

Comme le montre le graphique 3, après le vote de la loi de 1925 , les SARL ont très rapidement dominé les nouvelles entreprises enregistrées en France. La forme semble avoir été particulièrement attractive pour les sociétés avec un petit nombre d'associés mais dont les besoins en capitaux étaient plus importants que ceux de la plupart des sociétés en nom collectif. En 1927, par exemple, $57 \%$ des SARL créées avaient seulement deux associés, mais un capital moyen double de celui des sociétés de personnes créées la même année (SNC et commandites comprises). En revanche, le capital des SARL était moindre que celui des sociétés anonymes qui se formaient au même moment. En 1927, leur capital moyen représentait moins du quart de celui des nouvelles sociétés anonymes, et seules $10 \%$ des SARL avaient un capital supérieur à celui de la société anonyme moyenne. Le capital des nouvelles SARL était en moyenne de 100000 francs, quatre fois le minimum exigé par la loi. Plus précisément, si les SARL avaient pour la plupart un capital si faible qu'elles auraient sans doute été des sociétés de personnes sans la loi de 1925, celles ayant beaucoup de capitaux (disons le cinquième le plus capitalisé) auraient très probablement été des sociétés anonymes ${ }^{52}$.

\section{L'Allemagne, le Royaume-Uni et la France}

Pour comparer les expériences de ces trois pays, nous devons choisir un intervalle de temps uniforme et tenir compte du fait que nous n'avons pas partout accès aux mêmes données. Nous mesurons la diffusion de la nouvelle forme à partir de l'année où la législation a autorisé les SARL en employant deux étalons différents. Le premier, que nous pouvons calculer pour chacun des trois pays, est la proportion de sociétés par actions qui ont été formées en SARL (plutôt qu'en sociétés anonymes ou commandites par actions). La seconde, que nous ne pouvons estimer que pour la France et l'Allemagne, est la part de toutes les compagnies multi-propriétaires organisées en SARL.

Notre première mesure suggère que les expériences prussiennes, britanniques et françaises se ressemblent (voir graphique 6). Après le vote des lois autorisant la nouvelle forme, les nouvelles sociétés par actions sont devenues pour une très grande part des SARL. Cette diffusion rapide cache néanmoins des différences substantielles entre les trois pays. En France, la création d'une société anonyme était beaucoup moins coûteuse qu'en Allemagne, et l'arrivée de la SARL a donc eu un effet plus important sur le nombre de nouvelles sociétés anonymes. De même, ces dernières étaient bien plus fréquentes au Royaume-Uni qu'en France, et, comme nous l'avons vu, la SARL les a pratiquement éliminées. 
Graphique 6 - Part des SARL dans les nouvelles sociétés de capitaux

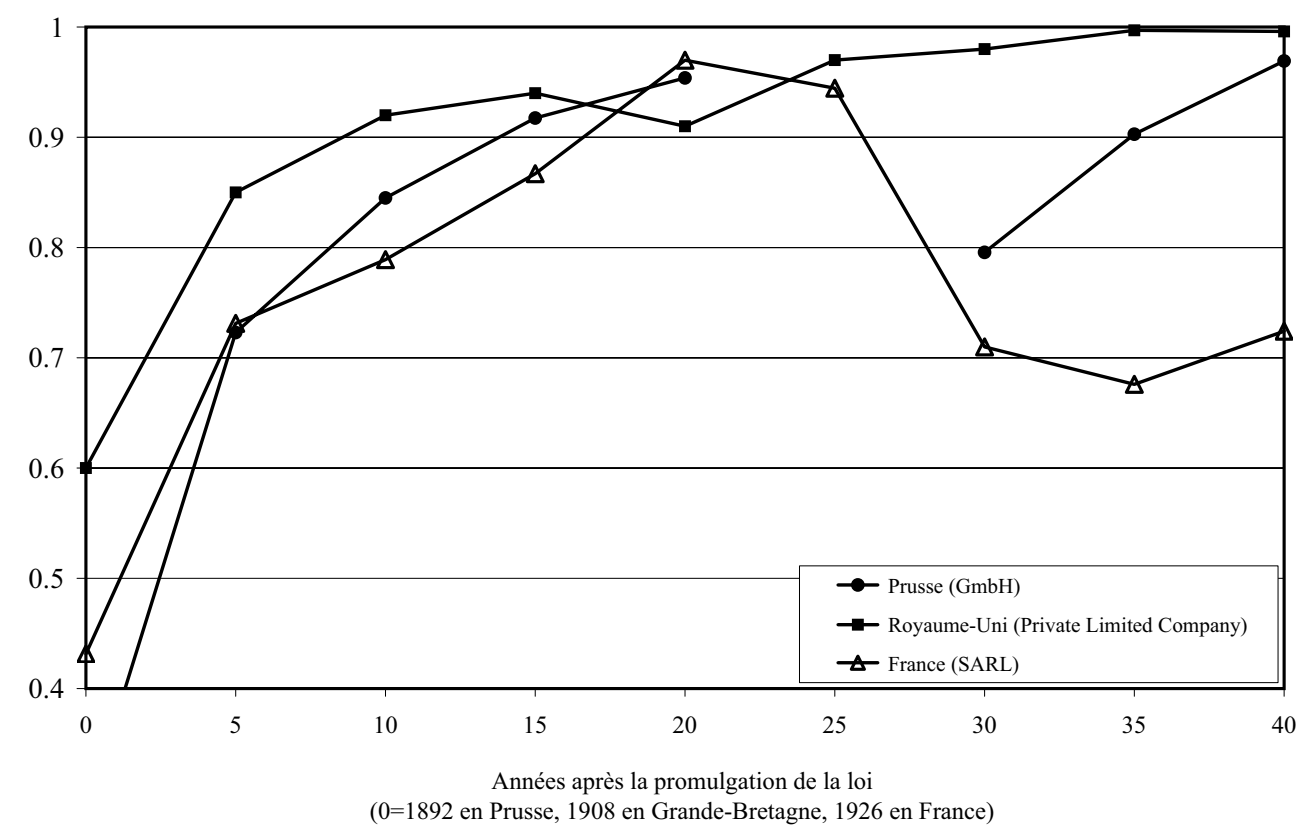

Sources: Voir graphiques 3, 4 et 5.

Quoique les données nécessaires manquent pour inclure le cas britannique dans le graphique 7, nous savons que l'arrivée de la SARL a également réduit le nombre de sociétés de personnes (SNC et commandites simples), mais sans doute pas autant qu'en France. Ces sociétés ont également mieux résisté à l'introduction des SARL en Allemagne qu'en France. L'explication tient peut-être, en partie, au fait que les GmbH disposaient d'un capital minimum plus élevé que les SARL. Elle tient sans doute aussi à un dispositif propre à la loi allemande. Les membres de n'importe quelle société avaient la possibilité de nommer un Prokurist (fondé de pouvoir). Ce dernier pouvait agir seul au nom de la société, donnant à l'association ordinaire ( $\mathrm{OHG}$ ) ou à la commandite simple (KG) certains des avantages de la gérance concentrée des sociétés par actions ${ }^{53}$. Le Prokurist permettait ainsi aux sociétés de personnes de résoudre un des problèmes de cette forme d'organisation en concentrant tous les pouvoirs sur un seul et même individu.

En Allemagne, la proportion de commandites change peu quand est introduite la GmbH, alors qu'elle s'effondre en France avec l'arrivée de la SARL. Ce résultat est moins surprenant qu'il n'y paraît si l'on considère la position des gérants. Comme leur part de capital ne leur donnait pas le pouvoir, ils étaient

53 - Otto EgGert, Die Prokura nach dem Handelsgesetzbuche vom 10. Mai 1897 unter besonderer Berücksichtigung der gegenüber dem Allgemeinen Deutschen Handelsgesetzbuche getroffenen Abänderungen, Leipzig, Julius Klinkhardt, 1902; Max Friedrich, Die Rechtsstellung des Prokuristen im geltenden Recht, Leipzig, Walter Wigand, 1905. 


\section{Graphique 7 - Part des SARL dans les nouvelles sociétés de commerce}

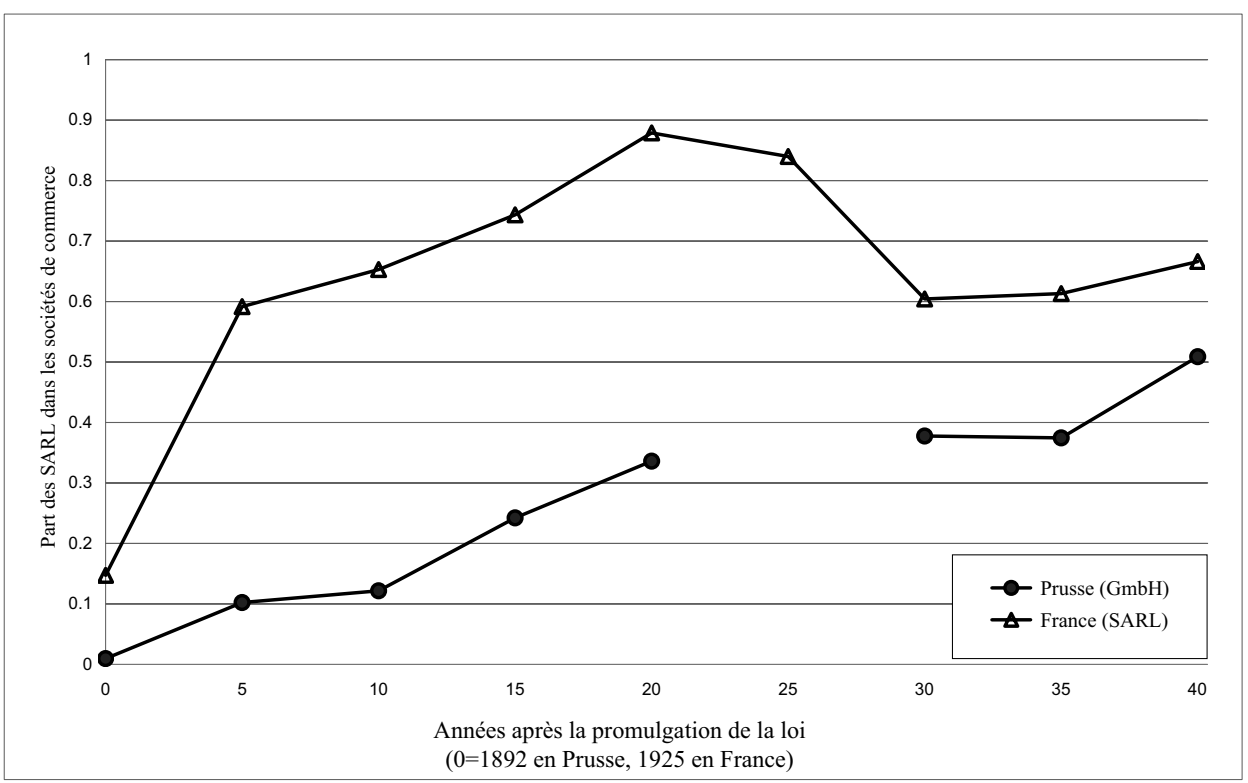

Sources: Voir graphiques 3 et 4.

toujours sous la menace d'un renvoi. Parce qu'en Allemagne, les actionnaires majoritaires avaient toute latitude pour écarter le gérant s'ils le souhaitaient, la GmbH était une moins bonne solution qu'une commandite simple si l'important était de pérenniser le contrôle par un ou deux gérants qualifiés mais n'ayant que peu de capital. En revanche les gérants français, dans une position semblable, pouvaient régler le problème dans une SARL en inscrivant leurs noms dans les statuts de l'entreprise. En ce sens, la SARL était un meilleur substitut à la commandite.

À en juger par l'exemple de ces trois pays européens, l'effet de l'introduction de la SARL sur le nombre de sociétés anonymes nouvelles dépendait du coût de création d'une société anonyme et de l'éventail des autres formes juridiques disponibles. Pour l'Allemagne et la France, la question mérite certes plus d'attention, mais l'effet de la SARL sur les autres formes juridiques semble avoir dépendu des dispositifs propres à la législation passée dans chaque pays. S'agissant du RoyaumeUni, il est en revanche plus délicat d'expliquer pourquoi les sociétés en nom collectif sont demeurées relativement populaires. La conversion en SARL peut avoir constitué un saut plus grand que sur le continent en raison du manque de formes intermédiaires. Il est également possible que le refus des tribunaux de common law d'intervenir dans les affaires internes des sociétés ait incité les individus qui craignaient de subir l'oppression des sociétaires majoritaires à ne pas renoncer à la capacité de se retirer en forçant une liquidation ${ }^{54}$. 


\section{Les États-Unis et le conservatisme de la common law}

En pratique, aux États-Unis, personne n'a eu accès à une forme de SARL avant la seconde moitié du $\mathrm{Xx}^{\mathrm{e}}$ siècle, quand les États ont commencé à modifier leurs lois sur les sociétés pour offrir aux PME la flexibilité contractuelle à laquelle les Européens étaient habitués. Il y eut, cependant, une tentative d'introduire une forme de SARL aux États-Unis avant que la GmbH soit autorisée en Allemagne. En 1874, la Pennsylvanie promulgua une loi qui donnait le droit à n'importe quel groupe de trois personnes ou plus occupées dans «n'importe quelles affaires ou métier reconnus" de s'organiser comme partnership association. Cette forme créait une entreprise reconnue comme personne morale, dont les parts n'étaient pas commercialisables, et où la responsabilité était limitée au «capital seul ». Des lois autorisant ces partnership associations furent ensuite adoptées dans le Michigan (1877), dans le New Jersey (1880) et l'Ohio (1881). La Virginie a également adopté une loi voisine en 1874, mais l'a abrogée en $1918^{55}$.

Les partnership associations, comme les SARL, étaient simplement créées en enregistrant les statuts auprès de la juridiction locale (le comté). Leur dissolution avant la fin du terme prévu n'était possible que par un vote de la majorité des associés en nombre et en parts, ce qui réduisait le risque de dissolution prématurée par rapport aux sociétés en nom collectif. Cependant, la capacité d'adopter des règles de vote qui protégeaient les actionnaires minoritaires variait légèrement d'un État à un autre. Alors que dans le New Jersey et l'Ohio les partnership associations pouvaient adopter les règles de vote qu'elles souhaitaient, un amendement à la loi de Pennsylvanie stipulait que les gérants devaient être élus à une majorité des parts. Néanmoins, les actionnaires minoritaires ont toujours eu la capacité de se retirer s'ils étaient en désaccord avec les décisions de la majorité. Tout détenteur de parts ne pouvait participer aux affaires de l'association qu'après accord d'une

55 - Les origines de la loi sont obscures mais son vote semble avoir été déclenché pendant les années 1870 en Pennsylvanie par un débat sur la libéralisation des sociétés anonymes. Plus tard, la Pennsylvanie a également voté une loi sur une autre forme de SARL, le «registered partnership», dont les dispositions étaient bien plus libérales. Edward H. WARren, Corporate advantages without incorporation, New York, Baker, Voorhis \& Co., 1929, chap. 4; Id., "Notes: Business associations in Pennsylvania ", University of Pennsylvania Law Review and American Law Register, 82, 1933, p. 153; George E. STRANSKY Jr., "The limited partnership association in New Jersey », Rutgers Law Review, 10, 1956, p. 701-702; James A. MatThEws Jr., «Comments: Business associations-registered partnership, partnership association or the corporation-selection of the suitable form in Pennsylvania», Villanova Law Review, 2, 1957, p. 386-387; Edward R. SCHWARTZ, "The limited partnership association-an alternative to the corporation for the small business with 'control' problems?", Rutgers Law Review, 20, 1965, p. 3033; Wayne M. Gazur et Neil M. Goff, «Assessing the limited liability company », Case Western Reserve Law Review, 41-2, 1991, p. 393-394. Ces lois et amendements passés avant 1899 sont disponibles sur microfilm : Session Laws of American States and Territories. Toutes les références à la législation spécifique ci-dessous se rapportent à cette collection et sont citées simplement à leur date. 
«majorité des membres en nombre et en part d'intérêts » et n'importe quel associé non admis aux affaires devait être remboursé de ses parts à un prix mutuellement convenu ou, à défaut d'accord, déterminé par le tribunal civil local (Court of Common Pleas) ${ }^{56}$.

Bien que l'adoption de la loi en Pennsylvanie ait amené une première vague de sociétés à s'organiser en SARL (y compris des entreprises célèbres comme Carnegie Steel Company Ltd.), cette forme ne s'est pas diffusée au-delà du groupe initial d'États et elle n'a été utilisée que durant une vingtaine d'années. En effet, les tribunaux de common law, dont on vante les mérites, ont tué cette première SARL. Par une série de décisions importantes, les tribunaux de Pennsylvanie ont établi que si les statuts enregistrés d'une partnership association étaient incorrects sur un point matériel, ou que si la liste du capital de l'association rendait «sa valeur difficile à juger» parce qu'elle agrégeait différentes catégories d'équipement, l'association n'était pas valable et ses membres étaient entièrement responsables des dettes de leur entreprise comme dans une société en nom collectif ${ }^{57}$. Ce faisant, les tribunaux invitaient les créanciers des associations insolvables à intenter des procès dans l'espoir qu'elles pourraient démontrer des erreurs ou des insuffisances dans les formalités d'enregistrement et annuler la responsabilité limitée.

Il est remarquable d'observer que, pour des sociétés anonymes, la règle était exactement inverse: leur existence légale était présumée. Les tribunaux ont justifié leur décision en relevant deux différences entre les partnership associations et les sociétés anonymes. Bien qu'en pratique les premières «aient été dotées de plusieurs dispositifs et capacités d'une société », dans une partnership association, à la différence d'une société anonyme, «aucun homme ne peut acheter l'intérêt d'un membre et dès lors participer aux affaires, à moins [d'être approuvé] par une majorité des membres en nombre et en valeur de leurs intérêts». De plus, les juges ont déclaré que l'État n'avait pas accordé de charte aux partnership associations; leurs privilèges étaient donc établis entièrement par les documents enregistrés. En conséquence, il était «loisible» pour un créancier qui portait plainte pour le paiement d'une dette «de rechercher un défaut mortel» dans les statuts «ou de montrer qu'une condition requise, bien que formellement indiquée, avait été faussement énoncée ${ }^{58}$.

La jurisprudence conservatrice de Pennsylvanie a été aggravée par la décentralisation du droit commercial aux États-Unis. Il pouvait donc y avoir des variations dans le traitement d'une affaire par les tribunaux de différents États. Dans un "Putting the corporation in its place», op. cit.

57 - Maloney v. Bruce, 94 Pa. 249 (1880); Eliot v. Himrod, 108 Pa. 569, 580-81 (1885); Appeal of Hite Natural Gas Co., 118 Pa. 436 (1888); Vanhorn v. Corcoran, 127 Pa. 255 (1889); Sheble v. Strong, 128 Pa. 315 (1889); Gearing v. Carroll, 151 Pa. 79 (1892).

58 - Eliot v. Himrod, 108 Pa. 569, 580-81 (1885). Des restrictions similaires ont pratiquement tué la société en commandite aux États-Unis; dans ce cas-ci, le tribunal de première instance avait essayé d'empêcher des associations de connaître un destin semblable en arguant que la législation avait prévu de les traiter comme des sociétés plutôt qu'en tant que sociétés en commandite. Mais cet avis fut cassé en appel. 
litige du Massachusetts, par exemple, une partnership association de Pennsylvanie fut tenue pour société en nom collectif et ses membres responsables sans limite. En outre, dans certains domaines, des juridictions fédérales ont aussi traité les partnership associations comme si elles étaient des sociétés en nom collectif ${ }^{59}$.

$\mathrm{Si}$ les entreprises britanniques n'ont pas eu à subir les incertitudes liées au fédéralisme, elles ont aussi souffert du conservatisme de la common law. L'hostilité pluriséculaire des tribunaux aux sociétés en commandite peut expliquer pourquoi une loi de 1907 autorisant cette forme a eu peu de conséquences ${ }^{60}$. En revanche, l'autre loi de 1907 créant les SARL (PLLC) s'est imposée parce qu'elle s'en tenait à une innovation modeste. En effet, elle stipule seulement que les PME étaient exemptes des règles onéreuses que le parlement avait imposées pour empêcher les abus dans les sociétés anonymes dont le capital était ouvert au public. Les entreprises qui se sont organisées en tant que SARL ont donc bénéficié d'un demisiècle de jurisprudence favorable sur les sociétés anonymes.

Pour des raisons analogues, les modifications apportées après 1945 par beaucoup d'États américains à leurs lois sur les sociétés ont plus favorisé l'essor des SARL que les lois sur les partnership associations. La volonté de changement semble alors liée à l'évolution relative de la fiscalité sur le revenu personnel et sur les sociétés ${ }^{61}$. Pour réduire leurs impôts, investisseurs et entrepreneurs ont choisi d'organiser leurs entreprises en sociétés anonymes plutôt qu'en sociétés en nom collectif (voir les tableaux 1 et 2). Ce glissement semble avoir créé un courant d'opinions favorables à des changements tendant à rendre les formes juridiques des sociétés plus appropriées aux PME ${ }^{62}$. La première réforme importante fut votée en Caroline du Nord en 1955. La nouvelle loi sur les sociétés de commerce comprenait plusieurs dispositions destinées aux petites sociétés avec peu de propriétaires, dont une déclarant que les accords pris par tous les actionnaires de ces sociétés, quelle qu'en

59 - Edwards v. Warren Linoline \& Gasoline Works, 168 Mass. 564 (1897); and Great Southern Fire Proof Hotel Co. v. Jones, 177 U.S. 449 (1900). Voir aussi E. H. WARrEN, « Notes: Business associations in Pennsylvania», art. cit., p. 153 ; G. E. STRANSKY Jr., «The limited partnership association in New Jersey ", art. cit., p. 709-710; E. H. Warren, Corporate advantages without incorporation, op. cit., p. 517-519.

60 - Seules 144 sociétés en commandite ont été formées l'année qui a suivi la promulgation de la loi, mais ce nombre déjà faible s'est ensuite vite réduit. Dans les années 19111920, il tombe à environ 50 par an, et à 37 seulement entre 1921 et 1930 . UK Board of Trade, General annual report under the Companies (Winding-up) Act of 1890, Londres, 19001921; UK Board of Trade, General annual report, Londres, 1922-1930.

61 - W. E. Brownlee, Federal taxation in America..., op. cit., p. 89-129.

62 - Au cours de ces années se multiplient les articles présentant de façon générale le cadre légal des entreprises, avec pour objectifs: (1) d'informer les avocats sur les articles qu'ils peuvent inclure dans les statuts des SA pour protéger les investisseurs, (2) d'inciter à une nouvelle législation qui augmenterait la flexibilité de la SA. Voir Forrest H. O'NEAL, "Giving shareholders power to veto corporate decisions: Use of special charter and by-law provisions ", Law and Contemporary Problems, 18, 1953, p. 451-472; Id., "Developments in the regulation of the close corporation », Cornell Law Quarterly, 50, 1965, p. 641-662; Id., "Close corporations: Existing legislation and recommended reform ", The Business Lawyer, 33, 1978, p. 873-888; Id., Close corporations: Law and practice, Chicago, Callaghan, 1958. 


\begin{tabular}{|c|c|c|c|c|}
\hline Industrie & Année & $\begin{array}{c}\text { Nombre } \\
\text { de sociétés } \\
\text { en nom collectif }\end{array}$ & $\begin{array}{l}\text { Nombre } \\
\text { de sociétés } \\
\text { anonymes }\end{array}$ & $\begin{array}{c}\text { Sociétés anonymes } \\
\text { en pourcentage } \\
\text { des sociétés de commerce }\end{array}$ \\
\hline Toute & $\begin{array}{l}1947 \\
1997\end{array}$ & $\begin{array}{r}888862 \\
1758627\end{array}$ & $\begin{array}{r}551807 \\
4710083\end{array}$ & $\begin{array}{l}0,38 \\
0,73\end{array}$ \\
\hline $\begin{array}{l}\text { Agriculture, } \\
\text { forêt et pêche }\end{array}$ & $\begin{array}{l}1947 \\
1997\end{array}$ & $\begin{array}{l}120402 \\
127060\end{array}$ & $\begin{array}{r}7329 \\
163114\end{array}$ & $\begin{array}{l}0,06 \\
0,56\end{array}$ \\
\hline Mines & $\begin{array}{l}1947 \\
1997\end{array}$ & $\begin{array}{l}13579 \\
28045\end{array}$ & $\begin{array}{r}8294 \\
32996\end{array}$ & $\begin{array}{l}0,38 \\
0,54\end{array}$ \\
\hline Construction & $\begin{array}{l}1947 \\
1997\end{array}$ & $\begin{array}{l}52592 \\
72098\end{array}$ & $\begin{array}{r}20287 \\
487783\end{array}$ & $\begin{array}{l}0,28 \\
0,87\end{array}$ \\
\hline Industrie & $\begin{array}{l}1947 \\
1997\end{array}$ & $\begin{array}{l}74978 \\
40022\end{array}$ & $\begin{array}{l}112184 \\
325045\end{array}$ & $\begin{array}{l}0,60 \\
0,89\end{array}$ \\
\hline $\begin{array}{l}\text { Transport, communication } \\
\text { et énergie }\end{array}$ & $\begin{array}{l}1947 \\
1997\end{array}$ & $\begin{array}{l}20776 \\
30917\end{array}$ & $\begin{array}{r}23729 \\
209402\end{array}$ & $\begin{array}{l}0,53 \\
0,87\end{array}$ \\
\hline $\begin{array}{l}\text { Commerce } \\
\text { de gros et de détail }\end{array}$ & $\begin{array}{l}1947 \\
1997\end{array}$ & $\begin{array}{l}372212 \\
173009\end{array}$ & $\begin{array}{rr}177297 \\
1149132\end{array}$ & $\begin{array}{l}0,32 \\
0,87\end{array}$ \\
\hline $\begin{array}{l}\text { Finance, assurance } \\
\text { et immobilier }\end{array}$ & $\begin{array}{l}1947 \\
1997\end{array}$ & $\begin{array}{r}87647 \\
974223\end{array}$ & $\begin{array}{l}151043 \\
744545\end{array}$ & $\begin{array}{l}0,63 \\
0,43\end{array}$ \\
\hline Services & $\begin{array}{l}1947 \\
1997\end{array}$ & $\begin{array}{l}130954 \\
310990\end{array}$ & $\begin{array}{r}45975 \\
1592854\end{array}$ & $\begin{array}{l}0,26 \\
0,84\end{array}$ \\
\hline Autres & $\begin{array}{l}1947 \\
1997\end{array}$ & $\begin{array}{r}15722 \\
2263\end{array}$ & $\begin{array}{l}5669 \\
5201\end{array}$ & $\begin{array}{l}0,27 \\
0,70\end{array}$ \\
\hline
\end{tabular}

Source: Susan B. CARTER et al., Historical statistics of the United States: Earliest times to the present, New York, Cambridge University Press, 2006, vol. 3, tabl. Ch 1-192.

Tableau 2 - Distribution des formes juridiques d'entreprises aux États-Unis, de 1949 à 2002

\begin{tabular}{cccc}
\hline \hline Année & $\begin{array}{c}\text { Sociétés } \\
\text { en nom collectif }\end{array}$ & $\begin{array}{c}\text { Sociétés } \\
\text { anonymes }\end{array}$ & $\begin{array}{c}\text { Sociétés à responsabilité limitée } \\
\text { (LLC) }\end{array}$ \\
1949 & 61 & 39 & - \\
1963 & 41 & 59 & - \\
1979 & 34 & 66 & - \\
1993 & 26 & 73 & 1 \\
2002 & 18 & 70 & 12 \\
\hline \hline
\end{tabular}

Sources: Susan B. CARTER et al., Historical statistics of the United States: Earliest times to the present, New York, Cambridge University Press, 2006, vol. 3, tabl. Ch 193-204; SOI Bulletin, diverses années.

Note: Les chiffres pour les SNC incluent les sociétés en commandite. Leur part des sociétés de commerce est passée d'environ 4\% en 1993 à environ $6 \%$ en 2002: ainsi le tableau minimise le déclin des SNC. 
soit la forme ou le but, «seront approuvé parce que [leur] effet est de rendre les actionnaires associés parmi eux-mêmes ». En d'autres termes, les propriétaires de petites sociétés anonymes pouvaient se comporter comme des membres d'une société en nom collectif. La même loi permettait d'intégrer dans les statuts d'une société le droit pour chaque actionnaire de demander la dissolution de la société «à volonté ou après l'occurrence d'un événement précis » ${ }^{63}$. Cette loi a donc permis aux membres des sociétés privées de limiter l'oppression des minoritaires, quitte à accepter d'augmenter le risque d'une dissolution prématurée.

Environ une douzaine d'États ont passé des lois semblables au cours des trois décennies suivantes. D'autres ont modifié leurs lois sur les sociétés de manière à augmenter la flexibilité de la forme et/ou ont donné aux juges le pouvoir d'intervenir (et même de dissoudre) dans les sociétés privées confrontées à des problèmes graves de dissension ou d'oppression de minorité. Néanmoins, dans les années 1980, certains juristes continuaient à exprimer l'opinion que beaucoup restait à faire aux États-Unis pour libérer « les sociétés privées des contraintes de la rigidité des lois sur les sociétés » et donner à des membres de ces sociétés plus de latitude pour mêler des attributs du nom collectif et des formes de sociétés ${ }^{64}$.

Ayant à nouveau bouleversé la donne fiscale, les baisses d'impôts réalisées sous la présidence de Ronald Reagan semblent avoir fourni l'impulsion pour des innovations législatives. Après qu'en 1988 le service des impôts ait décidé que des SARL (LLC) enregistrées par une loi du Wyoming seraient imposées comme des sociétés en nom collectif, d'autres États ont rapidement passé des lois semblables avec le but explicite de se conformer à la décision du Trésor ${ }^{65}$. Une deuxième vague de lois créant des sociétés en nom collectif à responsabilité limitée (LLP) a rapidement suivi ${ }^{66}$. Ceci a permis à tous les membres d'une société en nom collectif

63 - F. H. O'NeAL, «Developments in the regulation of the close corporation », art. cit., p. $647-648$.

64 - Kelvin H. DiCKINSON, "Partners in a corporate cloak: The emergence and legitimacy of the incorporated partnership ", American University Law Review, 33, 1984, p. 600. Voir aussi F. H. O'NeAL, "Developments in the regulation of the close corporation", art. cit.; Id., "Close corporations: Existing legislation and recommended reform ", art. cit.; Robert W. Hillman, «The dissatisfied participant in the solvent business venture: A consideration of the relative permanence of partnerships and close corporations ", Minnesota Law Review, 67, 1982, p. 1-88; Mark R. vON STERnBERG, «The close corporation's counterparts in France, Germany, and the United Kingdom: A comparative study », Hastings International and Comparative Law Review, 5, 1982, p. 291-322.

65 - La loi du Wyoming était presque une loi privée passée au profit d'une entreprise pétrolière particulière. Voir Robert R. Keatinge et al., «The limited liability company: A study of the emerging entity », The Business Lawyer, 47-2, 1991-1992, p. 381-384 ; Peter D. Hutcheon, «The New Jersey limited liability company statute: Background and concepts ", Seton Hall Legislative Journal, 18-1, 1993, p. 117-121 ; W. M. GAZUR et N. M. GoFF, «Assessing the limited liability company", art. cit., p. 390.

66 - Fallany O. Stover et Susan P. Hamill, «The LlC versus LlP conundrum: Advice for businesses contemplating the choice", Alabama Law Review, 50, 1999, p. 813-847. Bien que la loi initiale du Texas créant les LLP semble avoir été "une réponse aux pertes astronomiques menaçant avocats et comptables en raison du rôle de leurs associés dans les crises des caisses d'épargne de la fin des années 1980 », F. O. Stover et 
de limiter leur responsabilité pour les dettes de l'entreprise en enregistrant une notice spécifique. Bien que LLC et LLP soient à bien des égards semblables, les deux formes diffèrent par la manière dont elles équilibrent dissolution prématurée et oppression des minoritaires. Les LLP sont plutôt des sociétés de personnes desquelles les membres peuvent se retirer facilement. Les LLC sont plutôt des sociétés privées : à moins que les statuts prévoient explicitement une option de retrait, le capital des membres reste inclus dans l'entreprise tant que les dissensions internes ou l'oppression de minoritaires ne justifient pas l'intervention des tribunaux ${ }^{67}$. Ensemble, ces nouvelles formes ont élargi les choix contractuels aux États-Unis. Allant plus loin, des lois votées à la même époque ont encore étendu l'éventail des options. La loi de 1988 du Delaware sur les statutory business trusts a donné une liberté contractuelle pratiquement complète au monde des affaires pour l'organisation des entreprises. La législation n'a pas même indiqué de disposition type ${ }^{68}$.

On manque de données quantitatives sur le nombre d'entreprises formées comme SARL pendant le dernier tiers du $\mathrm{Xx}^{\mathrm{e}}$ siècle ou qui ont tiré profit de la flexibilité contractuelle accrue offerte par beaucoup d'États quand ils ont modifié leurs lois. Nous savons, cependant, que la proportion de sociétés à propriétaires multiples organisées comme sociétés en nom collectif est passée de $60 \%$ en 1949 à $34 \%$ en 1979 (voir le tableau 2). Bien qu'on puisse déduire de ce déclin que les entreprises ont adopté la SARL dès que la loi l'a permis, il se pourrait également qu'il reflète le traitement fiscal plus favorable octroyé aux sociétés pendant ces années. Nous savons aussi que les nouvelles LLC ont connu un grand succès à la fin du siècle. Selon le service des impôts, en 1993 (la première année pour lequel les données sont disponibles) il n'y avait environ que 17000 LLC aux États-Unis, mais près de 350000 en 1997 et plus de 946000 en 2002. Il n'y a aucun moyen de connaitre la proportion de nouvelles entreprises s'organisant en LLC, mais en 2002 celles-ci constituaient $12 \%$ de toutes les entreprises à propriétaires multiples aux États-Unis, contre moins de $1 \%$ en 1993. Ce gain semble s'être produit d'abord aux dépens des sociétés en nom collectif, dont la proportion a diminué de $22 \%$ en 1993 à $12 \%$ en 2002 (la part des sociétés en commandite est passée d'environ 4 à $6 \%$ ). En revanche, la proportion des entreprises à propriétaires multiples organisées en sociétés n'a guère baissé (de $73 \%$ en 1993 à $70 \%$ en 2002). Ce déclin relativement faible des sociétés suggère que les changements juridiques introduits par les États après la Deuxième Guerre mondiale ont considérablement

S. P. Hamill suggèrent que la diffusion rapide de la forme à d'autres États est plutôt due à des considérations fiscales.

67 - Sandra K. Miller, «What remedies should be made available to the dissatisfied participant in a limited liability company? ", American University Law Review, 44, 1994, p. 465-536; Carter G. BISHOP, « Unincorporated limited liability business organizations: Limited liability companies and partnerships ", Suffolk University Law Review, 29, 1995, p. 985-1058; Larry E. RibSTEIN, « Limited liability companies: Possible futures for unincorporated firms ", University of Cincinnati Law Review, 64, 1996, p. 319-368; F. O. STOver et S. P. Hamill, "The LLC versus LlP conundrum... », art. cit.

68 - Voir H. Hansmann, R. KraAkman et R. SQuire, "Law and the rise of the firm ", art. cit., p. 1397. 
augmenté la liberté contractuelle, remédiant à la plupart des inconvénients qui avaient justifié la si longue popularité des sociétés en nom collectif.

\section{La flexibilité contractuelle des SARL}

Analysons les statuts des SARL pour comprendre leur succès. Commençons par le cas de trois SARL françaises enregistrées au tribunal de commerce de Paris les 8 et 9 décembre $1932^{69}$. Ces exemples illustrent un aspect important des SARL qu'est leur capacité de répliquer d'autres formes d'organisations tout en les améliorant. En France, la source principale de cette flexibilité découle d'une clause de la loi de 1925 empruntée à la jurisprudence des commandites, qui indique que les gérants nommés dans les statuts ne sont pas révocables sans cause grave ${ }^{70}$.

Notre première société, Les Constructeurs, regroupait architectes et entrepreneurs du bâtiment et avait été formée pour réaliser des projets immobiliers. Les statuts désignaient deux des sociétaires pour être les gérants initiaux, mais stipulaient aussi qu'après six mois une assemblée générale choisirait les gérants effectifs. La gérance devait ainsi recevoir l'appui des propriétaires d'une majorité des parts. Comme les gérants initiaux ne possédaient que 392 des 1000 parts, les autres associés pouvaient les remplacer. Cette SARL était plutôt grande et, avec huit propriétaires et quatre fois le minimum exigé de capital, il aurait été malaisé d'agir à l'unanimité. Ses propriétaires ont donc choisi des statuts proches d'une société anonyme, sans cependant s'imposer le fardeau d'un conseil d'administration ou d'un conseil de surveillance.

Une deuxième SARL, Coupax, est enregistrée peu après. Formée pour fabriquer et commercialiser une machine pour la boucherie, elle avait trois propriétaires dont l'un fut nommé gérant. Bien que celui n'ait possédé que 18 des 40 parts, de fait, il avait tous pouvoirs, les autres étant des associés passifs. Ils contribuaient au capital de l'entreprise mais n'avaient aucun rôle dans la gestion. Les changements de statuts auraient exigé le consentement des propriétaires des 3/4 des parts, ce qui fait qu'en pratique le gérant avait aussi un droit de veto sur les statuts. Pratiquement, cette seconde SARL reproduit donc la structure d'une société en commandite avec l'avantage que la responsabilité de l'associé-gérant est limitée.

Le jour suivant est enregistrée notre troisième société, Stores et Enseignes, qui installait des équipements extérieurs pour les commerces de Paris. Elle réunissait deux associés, dont l'un détenait 3/5 des parts: selon les règles de vote des SARL, il aurait eu tous les pouvoirs. Cependant, les associés ont évité ce problème en nommant les deux propriétaires gérants statutaires. Puisqu'ils étaient tous deux inamovibles, ils devaient diriger la société par consentement unanime. Cette SARL était en fait une société en nom collectif dans laquelle le consentement des deux associés aurait été nécessaire pour emprunter au nom de la société. En sus, tous 
ses membres avaient droit à la responsabilité limitée et elle n'était pas sujette à la dissolution avant l'expiration de son terme. Plus surprenant, les statuts ont inclus une disposition complémentaire destinée à entrer en vigueur si l'un des propriétaires vendait ses parts à un parent. Dans ce cas, les décisions en assemblée se prendraient à la majorité des parts, sauf celle de changer les statuts qui nécessitait toujours l'unanimité ${ }^{71}$.

Comme le montrent ces trois exemples, grâce au simple changement d'un statut - le fait de nommer ou non un gérant -, les entrepreneurs français pouvaient employer la SARL pour obtenir des formes meilleures que les autres solutions juridiques possibles, évitant le fardeau des règles de la SA, éliminant la responsabilité illimitée qu'un ou plusieurs des associés devait accepter dans toute société de personne, et réduisant le problème de dissolution prématurée dans des SNC. Et leur marge de manœuvre était, en fait, plus grande encore.

Passons à un exemple allemand pour mettre l'accent sur certains autres aspects grâce auxquels la flexibilité contractuelle des SARL pouvait être étendue même si, on l'a vu, éviter le fardeau des règles de la société anonyme était encore plus important en Allemagne qu'en France. L'exemple provient d'une GmbH formée pour racheter une fabrique de passementerie à Nowawes (depuis rattaché à Potsdam) propriété d'Emmo Pechatscheck fils ${ }^{72}$. La mère de Pechatscheck, Helene, et deux autres personnes, Berthold Thon et Walter Vockel, ont formé la société pour racheter l'entreprise à Emmo fils. B. Thon investit 3/5 des 25000 marks du capital de l'entreprise, alors que W. Vockel et H. Pechatscheck y contribuent pour un cinquième chacun. Emmo père (mari d'Helene), qui n'était pas un associé, est nommé gérant. En s'organisant en $\mathrm{GmbH}$, les associés ont choisi de se protéger contre la dissolution prématurée, mais ils ont dû aussi s'inquiéter du risque d'oppression des minoritaires. Alors que la règle type aurait donné tout le pouvoir à B. Thon, puisqu'il possédait la majorité des parts, un article exigea le consentement unanime des propriétaires pour renvoyer le gérant. Par conséquent, Emmo père ne pouvait être renvoyé qu'avec l'accord de sa femme.

Les associés ont également ajouté des dispositions conçues pour protéger les membres de la famille contre la menace de la dissolution prématurée. S'ils souhaitaient se retirer, B. Thon et W. Vockel étaient obligés d'offrir leurs parts au père et au fils à un prix déterminé par une règle incluse dans le contrat. Cette disposition interdisait à des investisseurs extérieurs de vendre l'entreprise après en avoir pris le contrôle. En éliminant la capacité de W. Vockel et de B. Thon à forcer une liquidation, le pouvoir de négociation de la famille dans la société s'est donc trouvé renforcé. En échange de cette protection, les Pechatscheck ont offert à B. Thon

71 - Cette disposition ne devrait pas être interprétée comme créant des problèmes futurs pour le gérant minoritaire. En effet, il maintenait toujours la capacité de mettre un veto à n'importe quel changement de la structure de propriété (excepté une qui ferait suite à la mort de son associé).

72 - Emmo Pechatscheck Mechanische Posamentenfabrik für Möbel und Konfektion, Gesellschaft mit beschränkter Haftung, passé devant le notaire Raphael Josephsohn à Potsdam le 3 juin 1912. 
et W. Vockel une plus grande part des bénéfices de l'entreprise et ont garanti leurs investissements. L'article 4 indique que le capital investi sera rémunéré par un dividende de $5 \%$, ce qui est courant dans les GmbH. L'article 7 accorde en outre 2/5 des bénéfices supplémentaires à $\mathrm{B}$. Thon et à $\mathrm{H}$. Pechatscheck chacun. H. Pechatscheck et son mari garantissent également que les profits de l'entreprise devront être suffisants pour maintenir la valeur des investissements de B. Thon et de W. Vockel à leur valeur initiale et pour leur payer ce qui pourrait leur être dû. Helene n'aurait donc droit a rien si la firme n'était pas profitable ${ }^{73}$.

En principe, il aurait été possible d'écrire les statuts d'une société anonyme avec les mêmes dispositifs contractuels que l'exemple ci-dessus, mais cela aurait été plus compliqué et plus coûteux. En particulier, le contrat aurait dû dériver du contrat type d'une société anonyme et il aurait fallu recruter plusieurs autres investisseurs extérieurs pour arriver au minimum de sept actionnaires. Une société en commandite avec B. Thon et W. Vockel en tant que commanditaires aurait pu équivaloir à la $\mathrm{GmbH}$ du point de vue des associés, mais elle aurait laissé les Pechatscheck avec une responsabilité illimitée.

Dans cet exemple (comme dans beaucoup d'autres), les associés ont adopté des statuts qui ont rendu leur société tout à fait différente de ce qu'elle aurait été si elle avait suivi les dispositions type de la loi $\mathrm{GmbH}$ ou si elle s'était organisée en société en nom de personnes ou en société anonyme. Bien que des règles tout aussi complexes pour la distribution des bénéfices aient pu être adoptées dans une société en nom collectif ou même dans une société anonyme, les restrictions à la dissolution n'auraient pas été compatibles avec une société en nom collectif. Il n'aurait pas non plus été possible de protéger le gérant d'une société anonyme contre son renvoi.

Les organisateurs de PLLC en Royaume-Uni ont également tiré parti de la capacité de limiter des transferts de leurs parts. En 1927, par exemple, une famille britannique a formé une nouvelle société, Thomas Mitchell \& Sons (Guildford) Ltd., pour structurer ses affaires de fabrication de brique et de tuile ${ }^{74}$. La compagnie avait un capital de $22000 £$ divisé en 22000 parts de $1 £$ chacune. Quatre membres de la famille Mitchell ont reçu chacun un quart des parts. La famille a accepté la plupart des articles types du tableau A de la loi sur les sociétés mais en a néanmoins amendé quarante-cinq. La plupart des articles rejetés traitaient des assemblées de gérants et des actionnaires ainsi que des procédures pour nommer les gérants. Une addition notable aux statuts permettait aux gérants de refuser une cession de parts si l'associé vendeur ne leur démontrait pas que l'acheteur était une personne responsable ou s'ils ne trouvaient pas le transfert dans l'intérêt de la compagnie. Cette disposition leur donnait le pouvoir de s'assurer que la famille aurait le contrôle de la compagnie.

73 - Pechatscheck père a dû autoriser sa femme à accepter toutes les clauses du contrat. Ceci soulève un ensemble de questions complexes sur la propriété matrimoniale qu'on ne peut examiner ici.

74 - National Archives of the United Kingdom, BT 31/29949/224123. 
Un autre exemple britannique - celui d'une fabrique de métal, la N. C. Zinc Oxide Company - montre comment la décision de créer une SARL plutôt qu'une SA a donné aux propriétaires la liberté de contrat nécessaire pour résoudre leurs problèmes ${ }^{75}$. N. C. Zinc Oxide n'a pas adopté les statuts type mais a créé les siens propres. Les parts ont été divisées en deux classes: les 47500 parts de la classe A avaient une valeur nominale de $1 £$ tandis que les 50000 parts de la classe B valaient 1 shilling pièce. Les parts de la classe $\mathrm{A}$ avaient droit à un dividende prioritaire de $7 \%$. S'il y avait des bénéfices additionnels, $3 / 4$ iraient aux parts de la classe A et $1 / 4$ à celles de la classe B. Henry Edwin Coley, qui se désigne comme chercheur technique (technical investigator), reçut toutes les parts B en échange de l'apport à la compagnie d'un permis pour l'utilisation d'un brevet pour la fabrication du zinc. La banque Lloyds détenait $61 \%$ des parts A, le reste étant détenu par 36 autres actionnaires dont 25 avaient moins de $1 \%$ des parts. Chaque part A ou B ayant une voix, le pouvoir était donc donné à $\mathrm{H}$. Coley que les statuts nommaient aussi gérant à vie.

L'exemple illustre bien comment un entrepreneur qui voulait lever des capitaux substantiels auprès d'investisseurs externes sans perdre le pouvoir pouvait utiliser la flexibilité contractuelle offerte par les SARL. Les statuts ont également permis à un financier extérieur d'acquérir un actif financier qui mêlait des caractéristiques de l'obligation (par le dividende fixe de son action privilégiée) et de capitaux propres (parce que les mêmes actions avaient droit à $75 \%$ du profit résiduel). Les statuts de N. C. Zinc Oxide incitaient en outre l'entrepreneur (H. Coley) à obtenir un niveau élevé de bénéfices parce qu'il ne touchait un profit qu'après que la société ait gagné au moins 3325 £. Le gros investissement de la banque lui donnait de par la loi un droit de veto sur certaines décisions et, en l'absence de clauses contraires, elle avait la capacité de vendre dans le futur ses parts à des tiers ${ }^{76}$.

Aux États-Unis, les entrepreneurs n'ont pas eu accès à de tels choix jusque bien après la Deuxième Guerre mondiale. Néanmoins, les statuts des partnership associations, apparus après que la Pennsylvanie ait voté en 1874 une loi les autorisant, suggèrent que les Américains voulaient ainsi structurer leurs sociétés selon des modalités que ni les sociétés en nom collectif ni les sociétés anonymes ne permettaient. Ainsi, les organisateurs de la Cecil Paper Co. Ltd., sans doute beaucoup plus inquiets de l'oppression des minoritaires que du risque de dissolution prématurée, ont choisi un terme légèrement en dessous de trois ans et ont établi des sauvegardes additionnelles dans leurs statuts. Les actionnaires devaient se réunir deux fois par an pour approuver les comptes de la société et pour évaluer la valeur de l'entreprise et des parts de chaque membre. S'il y avait une divergence, la valeur de l'entreprise devait être décidée par un vote où chaque membre de l'association aurait une voix. Pour d'autres questions, telles que l'élection des gérants, les votes se feraient à la majorité de la valeur des parts. Les investisseurs

75 - National Archives of the United Kingdom, BT 31/32835/222120.

76 - En principe presque toutes ces clauses auraient pu être adoptées par une société anonyme, mais il est probable que d'autres acteurs du marché financier (agents de 
minoritaires de la Cecil Paper Co Ltd. se satisfaisaient de laisser la majorité diriger la société, mais ils voulaient être sûrs d'obtenir leur part des bénéfices ${ }^{77}$.

En revanche, les investisseurs minoritaires de la Carnegie Steel Company Ltd. craignaient plus une dissolution prématurée que l'oppression des minoritaires quand ils ont organisé leur firme en 1892. Quelques années avant, Andrew Carnegie avait été si gravement malade qu'il avait failli mourir, et ses associés s'étaient alors aperçus que satisfaire ses héritiers pourrait provoquer une faillite. La solution qu'ils avaient conçue était d'ajouter une clause aux statuts donnant aux associés le droit de racheter les parts de Carnegie à leur valeur comptable sur une période de quinze ans. Tout associé qui souhaitait quitter la société était soumis aux mêmes règles; il devait vendre ses parts à leur valeur comptable (avec des payements fractionnés sur une période qui dépendaient de leur importance). Naturellement, les investisseurs minoritaires auraient pu se protéger contre la dissolution prématurée en organisant l'entreprise en société anonyme, mais Carnegie n'y était pas disposé. Il voulait pouvoir décider qui serait membre de la société (en récompensant les gérants dévoués avec des parts et en se débarrassant des associés qui ne partageraient pas sa stratégie). C'est ainsi qu'il a imposé le fameux contrat «blindé » : tout membre de la société pouvait être forcé de vendre ses parts à la compagnie suite à un vote en ce sens des $3 / 4$ des associés en nombre et en valeur des parts. Carnegie a alors utilisé son pouvoir pour s'assurer que la valeur comptable de la compagnie restait bien au-dessous de sa valeur marchande, ce qui donna à l'accord une portée considérable ${ }^{78}$.

Avec la fin des partnership associations, les Américains ont perdu jusqu'à la fin $\mathrm{du} \mathrm{Xx}^{\mathrm{e}}$ siècle le gros de leur capacité à équilibrer dissolution prématurée et oppression des minoritaires et à contrôler l'identité des membres d'une société anonyme. Entrepreneurs et investisseurs aux États-Unis pouvaient éviter le danger de la dissolution prématurée en organisant des sociétés anonymes mais, dans la plupart des États, ils n'avaient pas beaucoup de marge pour réduire le risque d'oppression des minoritaires ${ }^{79}$. En 1945 encore, la cour d'appel de l'État de New York a invalidé les statuts d'une société exigeant le consentement unanime des actionnaires pour l'élection des directeurs parce que «l'État, accordant aux individus le privilège de limiter leurs responsabilités en se formant dans une entité séparée et distincte des personnes qui la possède, demande en retour que l'entité prenne une forme prescrite et se conduise dans ses procédures selon des règles fixes ${ }^{80}{ }$.

77 - Philadelphia City Archives, articles de l'Association of the Cecil Paper Co. Ltd., 13 novembre 1881, Limited Partnership, vol. 5 (LP5), Partnership Books, 1836-1955. 78 - Joseph F. Wall, Andrew Carnegie, New York, Oxford University Press, 1970, p. 491493; Harold C. Livesay, Andrew Carnegie and the rise of big business, éd. par O. Handlin, Boston, Little/Brown, 1975, p. 171-172; James Howard BRIDGE, The inside history of the Carnegie Steel Company: A romance of millions, Pittsburgh, University of Pittsburgh Press, 1991, p. 336-338.

79 - Pour plus de détails, voir N. R. Lamoreaux et J.-L. Rosenthal, «Legal regime and contractual flexibility... », art. cit., voir aussi T. W. Guinnane $e t$ al., «Putting the corporation in its place", op. cit.

80 - Benintendi v. Kenton Hotel, 294 NY 112, 118 (1945). 
Dans les deux décennies suivantes, on l'a vu, beaucoup d'États ont mis à jour leurs lois sur les sociétés pour leur donner plus de flexibilité contractuelle. Un membre de la commission qui rédigea la nouvelle loi pour le Connecticut déclara que c'était précisément ce genre de rigidité que le projet de loi voulait surmonter. Après avoir rappelé un exemple similaire où les tribunaux du Connecticut avaient invalidé un ensemble de statuts de sociétés écrits pour prévenir l'oppression des minoritaires, le témoin loua la nouvelle loi parce qu'elle incluait des « dispositions par lesquelles les [statuts] ${ }^{81}$ puissent requérir... plus qu'une majorité simple pour n'importe quelle décision ou pour la détermination de n'importe quelle question liée aux affaires de l'entreprise ». «La chose la plus attractive » de la loi était «cette flexibilité» qui «permet aux petites gens d'établir une société et de la conduire de la manière qu'elles prévoient sans sacrifier en quoi que ce soit les droits des créanciers ou les droits que ces gens se donnent entre eux ${ }^{82}$.

L'histoire de la SARL n'est pas conforme à l'idée courante selon laquelle la comparaison entre les pays de common law et ceux qui ont des codes civils ou commerciaux serait favorable aux premiers. Ni la chronologie de la législation autorisant les SARL, ni la rapidité de leur diffusion ne conforte ces visions manichéennes. L'Allemagne, pays de code, a agi la première, mais les firmes allemandes n'ont tiré parti de la nouvelle forme que lentement. Quand le Royaume-Uni, pays de common law, fit de même en 1907, la SARL a très vite remplacé la société anonyme mais les sociétés en nom collectif restèrent populaires. La France, pays de code, fut le troisième pays à adopter la SARL en 1925 et celle-ci est presque instantanément devenue la forme privilégiée, réduisant la formation de sociétés anonymes et éliminant presque entièrement les commandites simples et les sociétés en nom collectif. Les ÉtatsUnis enfin, pays de common law pourtant, n'ont pas mis à la disposition des entreprises une forme de SARL avant la deuxième moitié du $\mathrm{XX}^{\mathrm{e}}$ siècle. Depuis, le pays semble l'avoir adoptée à l'exclusion virtuelle de différentes formes de sociétés de personnes.

Une grande part des décalages dans la chronologie de ces pays est due aux événements et à des processus de path dependence propres à chacun. Les réformes allemandes de 1884, en réaction au «Gründerboom» de 1871-1873, avaient rendu la société anonyme si onéreuse qu'elles ont suscité de fortes pressions pour que soit fourni aux PME un autre choix. L'évolution du droit sur les sociétés au RoyaumeUni a rendu la société anonyme de plus en plus attrayante pour les PME dans la deuxième moitié du XIX ${ }^{\mathrm{e}}$ siècle jusqu'à ce que l'acte de 1900 , en réponse à des abus dans de grandes sociétés anonymes, rende la forme moins appropriée aux entreprises qui n'avaient pas l'intention d'être cotées en bourse. Comme en Allemagne, cette loi a poussé à la création d'une législation pour les SARL. En

81 - En anglais: the by-laws or the certificate of incorporation.

82 - Témoignage de Harry L. Nair, président du Committee of Corporations of the State Bar Association, devant le General Law Committee, 27 avril 1959: Connecticut General Assembly, Joint Standing Committee Hearings: General Law, Part 3, 1959. 
France, l'histoire fut très différente puisque c'est le retour de l'Alsace et de la Lorraine qui introduisit des GmbH. Aux États-Unis, il est probable que les taux d'imposition fiscaux relatifs ont encouragé des PME à se transformer en sociétés anonymes et ont poussé à des changements qui rendraient celles-ci plus attractives.

Dans tous ces cas, le succès des SARL remet en cause la supériorité supposée de la société anonyme. Même là où celle-ci était la plus populaire pendant la fin du XIX ${ }^{e}$ siècle (au Royaume-Uni et aux États-Unis), elle n'a jamais été adoptée par plus de la moitié des entreprises à propriétaires multiples. Les entrepreneurs étaient contraints de choisir entre deux formes de sociétés également imparfaites. C'est pourquoi ils ont exigé une forme intermédiaire comme la SARL qui offrait certains des avantages des sociétés anonymes sans les inconvénients.

Parce que les SARL ne pouvaient pas lever de capitaux en faisant appel au public, notre article s'est concentré sur leur utilité pour les PME. Mais il existe beaucoup d'exemples de grandes sociétés qui ont choisi d'être des SARL ou même des sociétés en commandite. Dans d'autre cas, des grandes entreprises ont modifié leurs statuts de société anonyme pour obtenir les dispositifs contractuels (classes d'action multiples, par exemple, ou avantages spéciaux pour les actionnaires importants) qui sont des amendements substantiels au format type porté aux nues dans la littérature. Bien qu'historiquement la société anonyme et les entreprises à grande échelle aient grandi ensemble, la première n'était pas nécessaire pour les secondes. $\mathrm{Si}$ Andrew Carnegie n'avait pas besoin de société anonyme pour établir la plus grande et la plus efficace aciérie des États-Unis, qui ne pouvait s'en passer?

Timothy W. Guinnane Yale University

Ron Harris

School of Law, Tel Aviv University

Naomi R. Lamoreaux UCLA/National Bureau of Economic Research

Jean-Laurent Rosenthal California Institute of Technology 


\begin{tabular}{|c|c|c|c|}
\hline \multicolumn{4}{|c|}{ Annexe - Glossaire des termes et abréviations } \\
\hline Pays & Forme & Nom complet & $\begin{array}{l}\text { Nom court } \\
\text { ou abréviation }\end{array}$ \\
\hline Allemagne & Société anonyme & Aktiengesellschaft & $\mathrm{AG}$ \\
\hline $\begin{array}{l}\text { États-Unis } \\
\text { Royaume-Uni }\end{array}$ & Société anonyme & & Corporation \\
\hline Allemagne & SARL & $\begin{array}{l}\text { Gesellschaft mit } \\
\text { beschränkter Haftung }\end{array}$ & $\mathrm{GmbH}$ \\
\hline Allemagne & Société en commandite simple & Kommanditgesellschaft & KG \\
\hline Allemagne & $\begin{array}{l}\text { Société en commandite } \\
\text { par actions }\end{array}$ & $\begin{array}{l}\text { Kommanditgesellschaft auf } \\
\text { Aktien }\end{array}$ & KGaA \\
\hline $\begin{array}{l}\text { États-Unis } \\
\text { Royaume-Uni }\end{array}$ & Société en commandite simple & Limited Partnership & LP \\
\hline $\begin{array}{l}\text { États-Unis } \\
\text { Royaume-Uni }\end{array}$ & SARL & Limited Liability Company & LLC \\
\hline Allemagne & $\mathrm{SNC}$ & Offene Handelsgesellschaft & $\mathrm{OHG}$ \\
\hline $\begin{array}{l}\text { États-Unis } \\
\text { Royaume-Uni }\end{array}$ & SNC & Simple Partnership & Partnership \\
\hline États-Unis & SARL & Partnership association & $\mathrm{PA}$ \\
\hline Royaume-Uni & Société anonyme & Public Limited Company & PLC \\
\hline Royaume-Uni & SARL & Private Limited Company & PLLC \\
\hline
\end{tabular}

